\title{
La (( nouvelle méthode implicite )) pour le calcul des tunnels dans les milieux élastoplastiques et viscoplastiques
}

D. BERNAUD

I. BENAMAR

G. ROUSSET

G. 3S/LMS

École Polytechnique

91128 Palaiseau Cedex
Le problème du creusement d'un tunnel est un problème de nature tridimensionnelle qui traduit un fort couplage entre deux structures très différentes: le massif avec l'excavation d'une part et le revêtement du tunnel d'autre part.

En vue d'une application souple, il est intéressant de mettre au point des méthodes simplifiées de dimensionnement des tunnels, qui permettent de réaliser les calculs en condition de déformation plane et de découpler ainsi le problème posé. Dans le cas simple où le problème admet la symétrie cylindrique, on montre que le paramètre clé qui conditionne l'interaction massif-soutènement est la convergence $U_{0}$ à la pose du soutènement (ou le taux de déconfinement à la pose $\lambda$.

L'objectif de cet article est d'étudier ce problème à l'aide de la méthode simplinée appelée $\alpha$ Nouvelle Méthode Implicite n, fondée sur les mémes principes généraux que ceux de la méthode classique convergenceconfinement, mais conduisant à une estimation plus précise du caractère couplé du problème d'interaction massif-soutènement. En particulier, cette méthode permet de tenir compte de la rigidité du soutènement (présence du soutènement déjà posé) dans le caicul de la convergence à la pose $\mathrm{U}_{\mathrm{f}}$.

Dans la première partie de l'article, on expose les derniers développements de la méthode concernant les massifs à comportements élastique et élastoplasique.

Dans la deuxième partie, on réalise l'extension de cette nouvelle méthode à un matériau viscoplastique de Tresca, en suivant la mème démarche établie pour les matériaux élastiques et élastoplastiques. Gråce à une analyse

adimensionnelle on met en évidence un paramètre fondamental du problème de creusement du tunnel dans un milieu viscoplasique: la vitesse réduite, qui dépend en particulier de la vitesse d'avancement de l'ouvrage et de la viscosité du massif. On montre, dans ce cas, que la convergence à I'instant de pose $\mathrm{U}_{0}$ dépend aussi de cette vitesse réduite.

La validation de la «Nouvelle Méthode implicite » est réalisée via des comparaisons avec des résultats de calcuis numériques axisymétriques sur une très large gamme de valeurs des paramètres indépendants du problème ef pour les diverses lois de comportement étudiées.

Cette méthode, souple d'emploi, fournit des solutions approchées d'une très bonne précision pour une étude géotechnique.

\section{The ((new implicit method)) for tunnel calculation: elastoplastic and viscoplastic rockmasses}

The tunnel excavation is a coupled three-dimensional problem concerning two differents structures: the rockmass and the lining

For a simple application it is interesting to develop simplified methods in onder to treat the probiem under plane strain assumption. When the problem of tunnel face adivance presents an axisymmetric geometry, we show that the major parameter governing the ground-interface-lining interaction is the convergence of the tunnel $\mathrm{U}_{n}$ at the moment of the lining installation

The simplified method studied here is the * New Implicit Method o that uses principles similar to those of the a Convergence-Confinement Method w, but it gives a better appreciation of the coupled behavior between the rockmass and the lining. For instance. in this new method the convergence $U_{b}$ depends aiso on the stiffness of the lining previously set.

In the first part of the paper, we present the recent improvements of the uNew Implicit Method $x$ for elastic and elastoplastic rockmasses.

The second part is devoted to the development of the new method to viscoplastic materials. A non dimensional analysis enables us to define a fundamental parameter of tunnel excavation in viscoplastic medium: the non dimensional funnel advance rate concerning the rate of excavation and the rockmass viscosity. For viscoplastic rockmass the closure $\mathrm{U}_{0}$ depends on this non dimensional tunnel advance rate. The validity of the $\mathrm{kNew}$ Implicit Method o has been checked for the whole independents parameters of the problem.

This method is of very simple urilisation and its results are in good agreement with the FEM numerical results. Its accuracy is quite enough for a geotechnical study. 


\section{Introduction}

Le problème du calcul du tunnel soutenu présente deux particularités essentielles. D'abord, c'est un problème tridimensionnel puisque la proximité du front de taille rend le problème non symétrique. Par ailleurs, c'est un problème d'interaction pour lequel le couplage entre le massif et le soutènement est important.

Dans un article récent (Bernaud et Rousset, 1992), nous avons bien montré que l'équilibre final du tunnel revêtu que souhaite étudier le concepteur, dépend de l'ensemble des paramètres du problème et que les méthodes de dimensionnement simplifiées doivent rendre compte de ce couplage avec soin.

Ainsi, l'approximation classiquement admise par la méthode convergence-confinement, selon laquelle le taux de déconfinement $\lambda_{0}$ ou la convergence $U_{0}$ au moment de la pose du soutenement, ne dépendent pas de la raideur du soutènement, conduit à une sous-estimation de la pression de soutènement à l'équilibre, qui peut être importante dans beaucoup de cas.

La Nouvelle Méthode Implicite (NMI) que nous avons proposée dans un article précédent, permet de considérer la dépendance de $\mathrm{U}_{0}$ (ou $\lambda_{\mathrm{p}}$ ) en fonction du comportement du soutènement déjà posé et de rendre compte de toute la complexité du couplage massif-soutènement.

Le but de cet article est de faire le point sur les derniers développements de cette méthode:

- en élasticité et plasticité, on propose, dans un souci de simplification, une nouvelle forme de la fonction de soutènement et une extension de la méthode à de nouveaux critères de plasticité ;

- dans la deuxième partie de l'article, on propose l'extension de la nouvelle méthode à un matériau viscoplastique de Tresca en suivant la même démarche établie pour les matériaux élastiques et élastoplastiques.

\section{2}

\section{Définition du problème de base}

On considère un tunnel profond de section circulaire (rayon $\mathrm{R}_{1}$ ) creusé dans un massif dont le comportement est homogène et isotrope (Fig. 1), soumis initialement au champ de contrainte géostatique:

$$
\underline{\sigma}_{0}=-\mathrm{P}_{\infty} \underline{\underline{1}} \text { avec } \mathrm{P}_{\infty}=\rho \mathrm{gz}
$$

La profondeur du tunnel est grande devant son rayon, de sorte que l'on peut négliger le gradient de la pesanteur dans la zone du massif proche du tunnel et assimiler pgz à $\rho \mathrm{gH}$ ( $\mathrm{H}$ est la profondeur moyenne du tunnel) dans cette zone.

Le soutènement du tunnel, assimilé à un anneau d'épaisseur e constante, a lui aussi un comportement homogène et isotrope ; il est posé à une distance $\mathrm{d}_{0}$ constante du front de taille. Le front de taille et l'extrémité du soutènement sont plans et verticaux.

Avec ces hypothèses d'étude, le problème admet la symétrie cylindrique.

Une autre symétrie, très utile à la simplification de l'étude, est souvent constatée: le problème est à déformation plane si les deux conditions suivantes sont vérifiées:

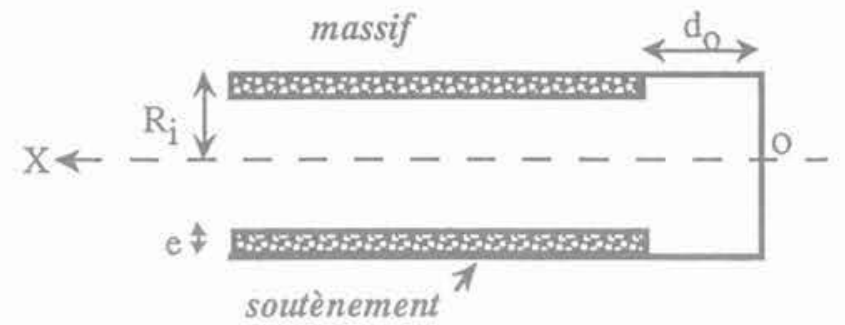

FIG.1 Modélisation axisymétrique du tunnel. Axisymmetric modelling of the tunnel.

- le front de taille est loin de la section d'étude; -la vitesse d'avancement $V$ du front et du soutènement est constante (cette dernière condition est nécessaire seulement lorsque les lois de comportement dépendent du temps, comme la viscoplasticité par exemple).

Dans ce cas, l'interaction entre massif et soutènement se traduit par un seul paramètre scalaire: la pression $P_{1}$ (poussée du terrain sur le revêtement ou pression de confinement). Le paramètre dual est la convergence de la paroi $U_{i}$, c'est-à-dire le déplacement radial normalisé de la paroi du tunnel, grandeur elle aussi scalaire $\left(U_{1}=-u(R) / R\right)$.

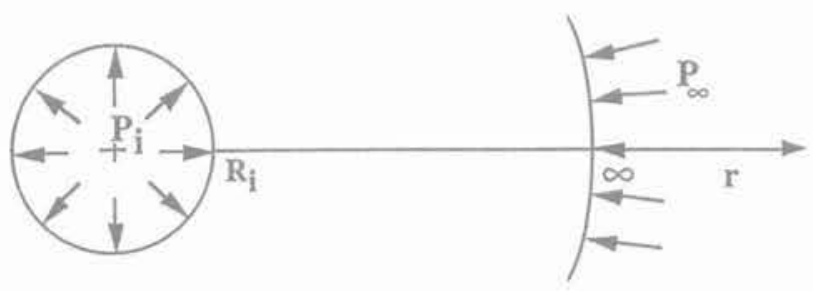

FIG. 2 Problème en déformation plane. Plane strain problem.

On peut maintenant définir deux courbes fondamentales (Fig. 3) indépendantes dans le diagramme $(\mathrm{P}, \mathrm{U})$ qui sont un «condensé » de l'information que l'on possède:

- la courbe de convergence CV: elle ne fait intervenir que la loi de comportement du massif; c'est la courbe

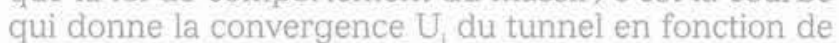
la pression $\mathrm{P}$ appliquée à la paroi, lorsque l'équilibre est atteint:

- la courbe de confinement CF : elle ne fait intervenir que la loi de comportement du soutènement; c'est la courbe qui donne la convergence $\mathrm{U}^{\mathrm{s}}$ de l'extrados de l'anneau en fonction de la pression $\mathrm{P}_{\text {}}$ appliquée au soutènement.

Au cours des phases préliminaires de creusement et de pose du soutènement, le problème est tridimensionnel (axisymétrique avec nos hypothèses) : la poussée $P_{1}$ du massif sur le revêtement et la convergence de la paroi $U$ dépendent de la distance $x$ de la section d'étude au front. Sur la figure $3, U_{\text {, représente la }}$ convergence très loin du front de taille pour le tunnel non soutenu.

Ce problème d'interaction de nature tridimensionnelle peut être traité par voie numérique (Bernaud, 1991; Corbetta, 1990; Hanafy et Emery, 1982; Pan et Hudson, 1989; Ranken et Ghaboussi, 1975). La méthode numérique utilisée dans cette étude est la "méthode d'activation/désactivation » développée dans le code aux éléments finis «GEOMEC91》)(Bernaud, 1991). 

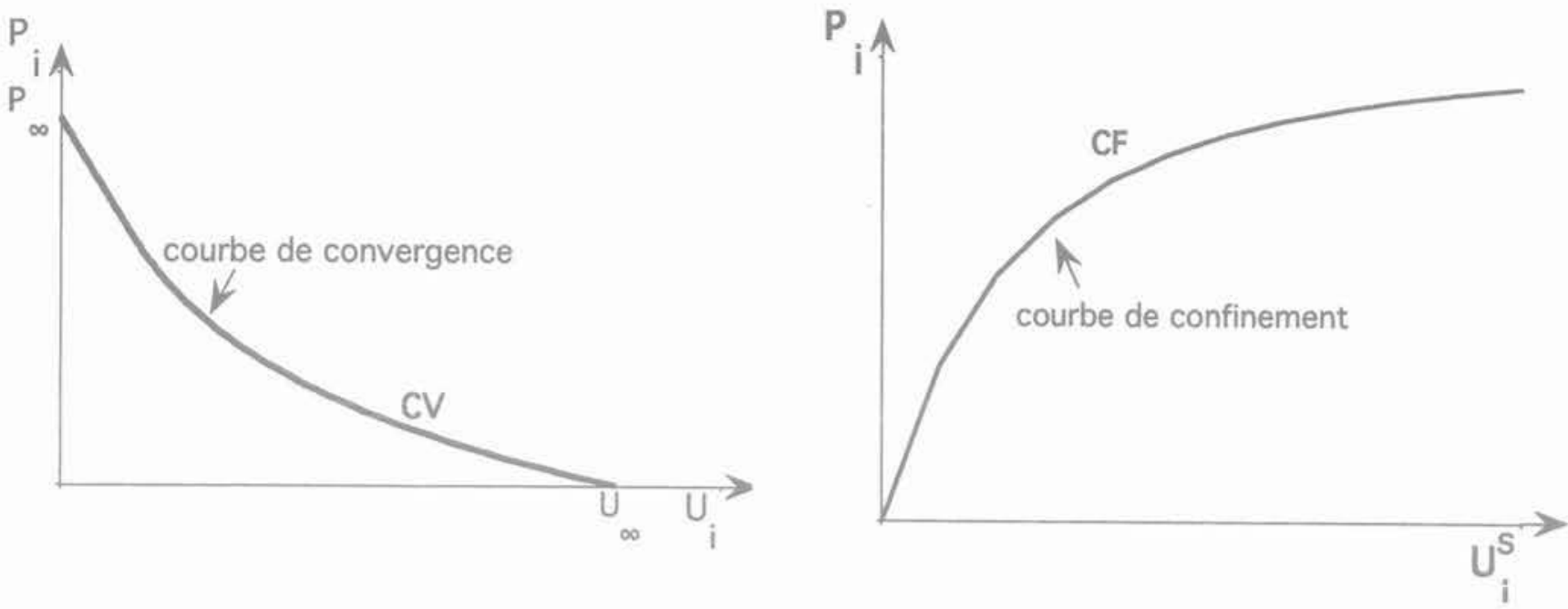

FIG.3 Courbes de convergence CV et de confinement CF, Convergence curve $\mathrm{CV}$ and confinement curve $\mathrm{CF}$.

Une analyse adimensionnelle du problème du tunnel soutenu dans un massif incompressible montre que le problème est à trois paramètres indépendants en élasticité et à quatre paramètres indépendants en plasticité pour un critère de Tresca ou Mises:

- élasticité:

$$
\mathrm{P}_{\infty}^{\prime}=\frac{\mathrm{P}_{\infty}}{\mathrm{E}} \quad \mathrm{K}_{\mathrm{s}}^{\prime}=\frac{\mathrm{K}_{\mathrm{s}}}{\mathrm{E}} \quad \mathrm{d}_{0}^{\prime}=\frac{\mathrm{d}_{0}}{\mathrm{R}_{\mathrm{i}}}
$$

- plasticité (Tresca, Mises):

$\mathrm{P}_{\infty}^{\prime}=\frac{\mathrm{P}_{\infty}}{\mathrm{E}} \quad \mathrm{K}_{\mathrm{s}}^{\prime}=\frac{\mathrm{K}_{\mathrm{s}}}{\mathrm{E}} \quad \mathrm{d}_{0}^{\prime}=\frac{\mathrm{d}_{0}}{\mathrm{R}_{\mathrm{i}}} \quad \mathrm{C}^{\prime}=\frac{\mathrm{C}}{\mathrm{E}}(3)$

avec $\mathrm{C}$ la cohésion, $\mathrm{E}$ le module d'Young du massif et $\mathrm{K}_{\mathrm{s}}$ la raideur du soutènement élastique.

\section{3}

\section{Méthode numérique}

La méthode de calcul des tunnels profonds en axisymétrie développée dans notre code de calcul éléments finis GEOMEC91 est la méthode « d'activation/désactivation $n$, qui permet de tenir compte du caractère tridimensionnel du problème de creusement d'un tunnel. Dans cette méthode, les séquences d'excavation et de pose du soutènement sont modélisées par le changement de la rigidité des éléments affectés à chaque phase de construction.

La figure 4 illustre de façon schématique notre modèle numérique pour étudier les tunnels profonds.

La méthode d'activation/désactivation résout directement le problème d'interaction massif-soutènement de façon couplée; cela signifie que les paramètres dimensionnants du tunnel (la convergence à l'équilibre $\mathrm{U}_{e q}$ et la pression à l'équilibre $\mathrm{P}_{\text {eq }}$ ) sont obtenus comme résultat direct du calcul à l'équilibre (Fig. 5). La connaissance préalable de la convergence à l'instant de la pose du soutènement $U_{0}$ n'est pas nécessaire, comme dans le cas de l'application de la méthode CV-CF.

Cette méthode numérique a été développée pour étudier des tunnels dans les milieux élastiques, élastoplastiques et viscoplastiques, avec des revêtements élastiques linéaires ou non. Son grand intérêt est que d'une part, elle tient compte du caractère tridimensionnel du problème, lorsqu'on se place dans des conditions d'axisymétrie, et d'autre part, elle permet de modéliser avec souplesse le phasage réel de construction de l'ouvrage.

Les calculs de tunnels en viscoplasticité à vitesse constante sont réalisés ici par une succession de pas d'excavations identiques (d'où, par abus de langage, vitesse constante). De façon pratique, pour obtenir la vitesse $\mathrm{V}$ choisie, on laisse, après chaque pas d'excavation, le massif fluer pendant l'intervalle du temps dt = pas $/ \mathrm{V}$.

D'autre part, en viscoplasticité, la longueur du maillage étant limitée par la taille d'un calcul par éléments finis, dans notre modèle la fin de la construction complète du tunnel ne correspond pas forcément à la stabilisation finale des mouvements. L'approche pour modéliser cette stabilisation, sans avoir à réaliser un tunnel de trop grande longueur est de laisser les déformations viscoplastiques se développer après le creusement entier du tunnel jusqu'à atteindre l'équilibre final recherché $\left(\mathrm{P}_{0,} \mathrm{U}_{\mathrm{e}}\right)$. On vérifie a posteriori que, puisque la longueur du tunnel est grande devant son rayon, la valeur du couple $\left(\mathrm{P}_{\mathrm{eq}}, \mathrm{U}_{\mathrm{eq}}\right)$ obtenu ne dépend pas de cette longueur et correspond donc à la solution cherchée: le point $\left(\mathrm{P}_{\mathrm{eq}}, \mathrm{U}_{\mathrm{eq}}\right)$ appartient bien à la courbe de convergence à long terme du massif.

\section{1}

\section{Calcul numérique du tunnel en plasticité}

Nous donnons ici l'exemple d'un calcul numérique d'un tunnel creusé à la profondeur de $200 \mathrm{~m}\left(\mathrm{P}_{-}=\right.$ $4 \mathrm{MPa}$ ) dans un massif élastoplastique parfait, obéissant à un critère de plasticité de Tresca $(E=500 \mathrm{MPa}$, $\nu=0,498, \mathrm{C}=1 \mathrm{MPa}$ ). Le soutènement est élastique, de raideur $\mathrm{K}_{\mathrm{s}}=1200 \mathrm{MPa}$, et il est posé à une distance $\mathrm{d}_{0}=2 / 3 \mathrm{R}_{\mathrm{s}}$ du front.

La figure 7 donne les profils de convergence en fonction de la distance au front pour chaque creusement. La convergence en paroi $\mathrm{U}_{0}$ au moment où est posé le soutènement (décompression du massif), à la distance $d_{0}=2 / 3 R$ du front, vaut $1,8 \%$.

La convergence finale à la stabilisation $U_{e q}$ vaut 


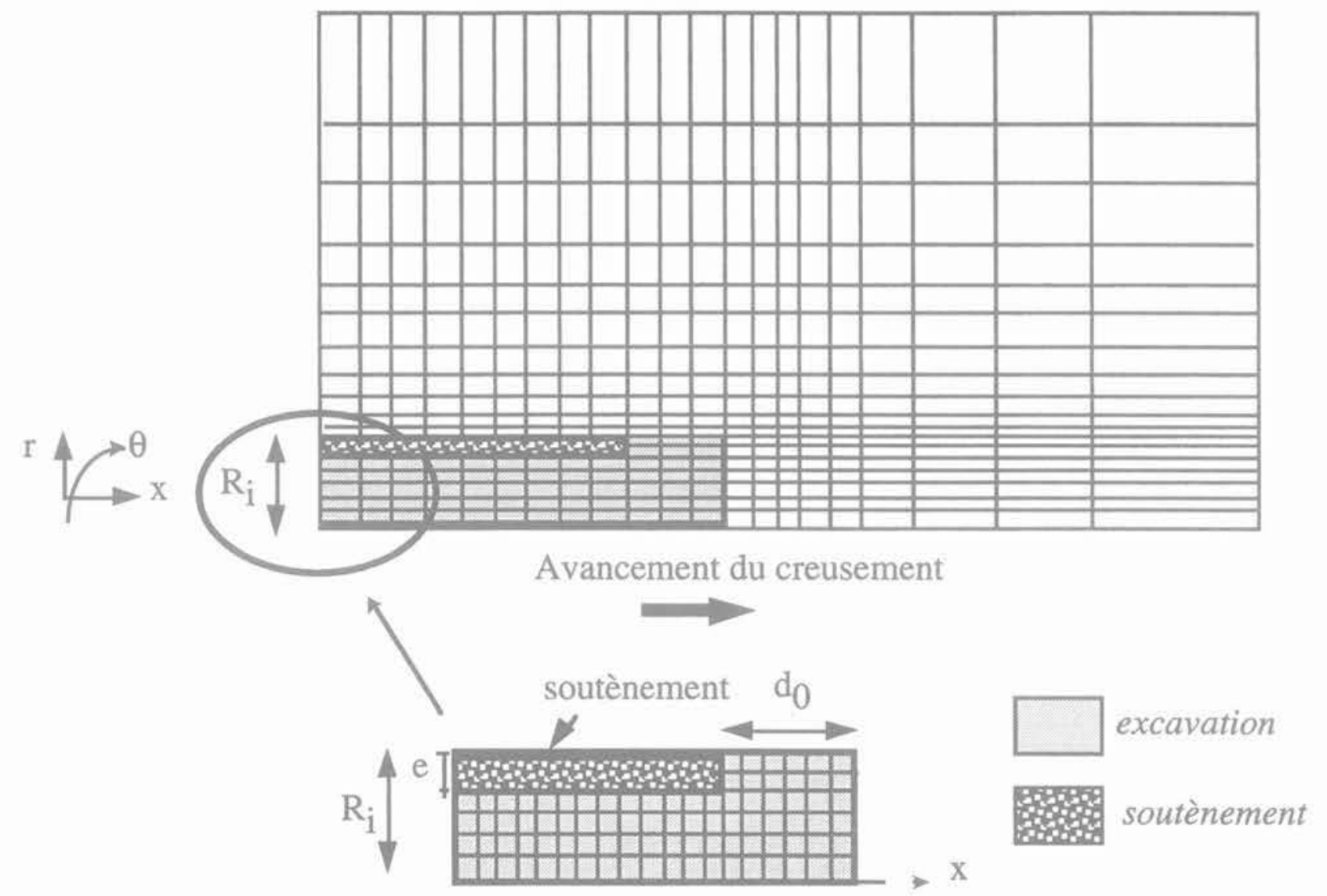

FG.4 Exemple type de maillage du modèle. Typical mesh of tunnel modelling

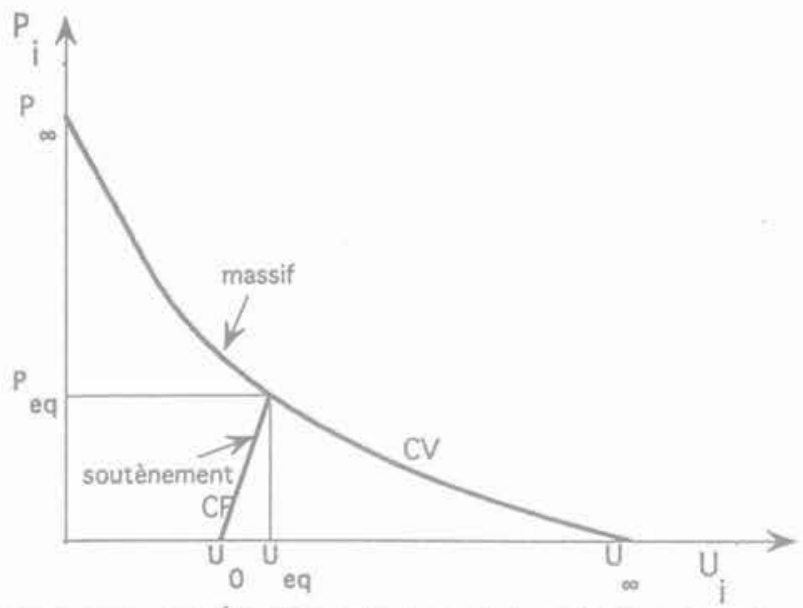

FG. 5 Équilibre du tunnel dans le diagramme $\mathrm{P}_{\mathrm{T}} \times \mathrm{U}_{\text {. }}$

Tunnel equilibrium in a diagram $\mathrm{P} \times \mathrm{U}$.

$1,90 \%$ pour les sections situées à une distance supérieure à $3 \mathrm{R}$ du front (zone d'influence du front); la convergence du soutènement est donc de $\mathrm{U}_{\mathrm{s}}=\mathrm{U}_{\mathrm{et}}-\mathrm{U}_{0}$ $=0,10 \%$. La pression à l'équilibre (poussée du massif sur le soutènement lorsque le front de taille est loin), vaut $\mathrm{P}_{\text {eq }}=1,16 \mathrm{MPa}$ (Fig. 6). Ce point $\left(\mathrm{P}_{\mathrm{eq}}, \mathrm{U}_{\mathrm{eq}}\right)$ est repéré sur la figure 8 et on peut constater que, comme prévu, le point d'équilibre obtenu numériquement appartient bien à la courbe de convergence du massif, ce qui constitue une validation des calculs puisque cette courbe est obtenue analytiquement.

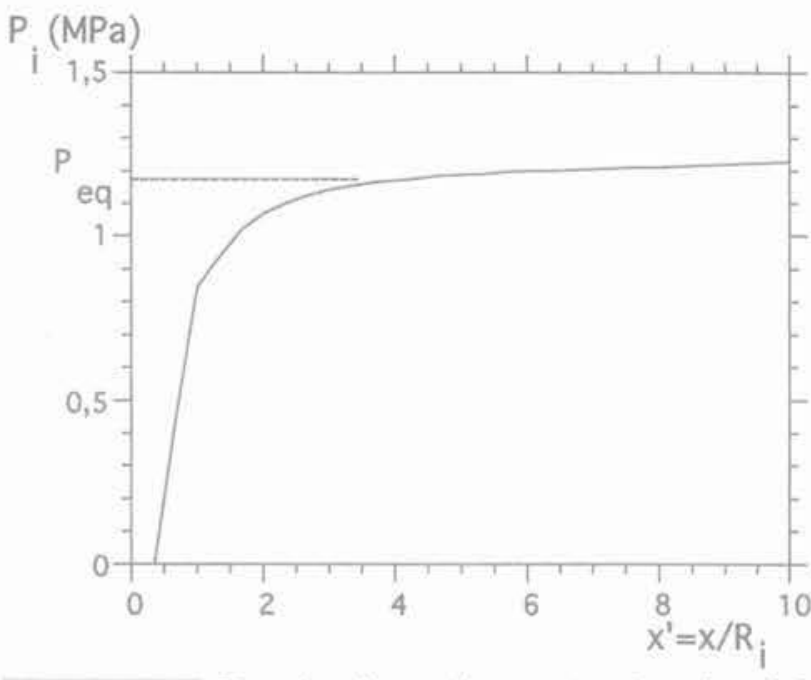

FIG.6 Pression du soutènement en fonction de la distance au front. Lining pressure versus distance to the tunnel face.

\section{2}

\section{Calcul approché par la méthode convergence-confinement}

On peut résoudre ce même problème par la méthode convergence-confinement (AFTES, 1983; Panet et Guellec, 1974).

La méthode convergence-confinement suppose implicitement que la valeur de $\mathrm{U}_{0}$ ne dépend que de la 


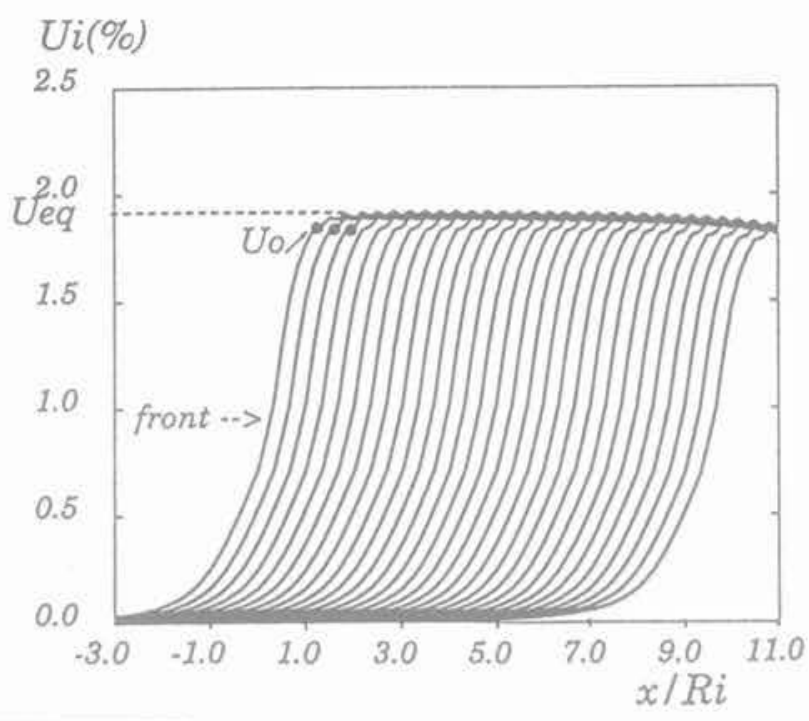

FIG.7 Convergence en fonction de la distance au front.

Convergence versus distance to the tunnel face.

distance $\mathrm{d}_{0}$ du front au soutènement et peut se calculer à partir de la courbe de convergence Uf(x) du tunnel non soutenu en fonction de la distance au front comme le montre le schéma de la figure 9 . Si l'on préfère, la méthode CV-CF propose une estimation simple du taux de déconfinement, qui pour une loi de comportement donnée, ne dépend que de $\mathrm{d}_{0}$.

Une première analyse critique approfondie de la méthode convergence-confinement a été réalisée dans Bernaud (1991) et poursuivie dans Bernaud et Rousset (1992).

A titre d'illustration on montre ici l'application de cette méthode au cas traité précédemment par voie numérique: le calcul avec la méthode CV-CF donne $\mathrm{U}_{0}=2,94 \%, \mathrm{P}_{\mathrm{eq}}=0,70 \mathrm{MPa}, \mathrm{U}_{\text {eq }}=3,0 \%$, soit une erreur de $40 \%$, ce qui est important, sur l'estimation du paramètre dimensionnant fondamental, qui est la pression à l'équilibre $\mathrm{P}_{\mathrm{eq}}$.

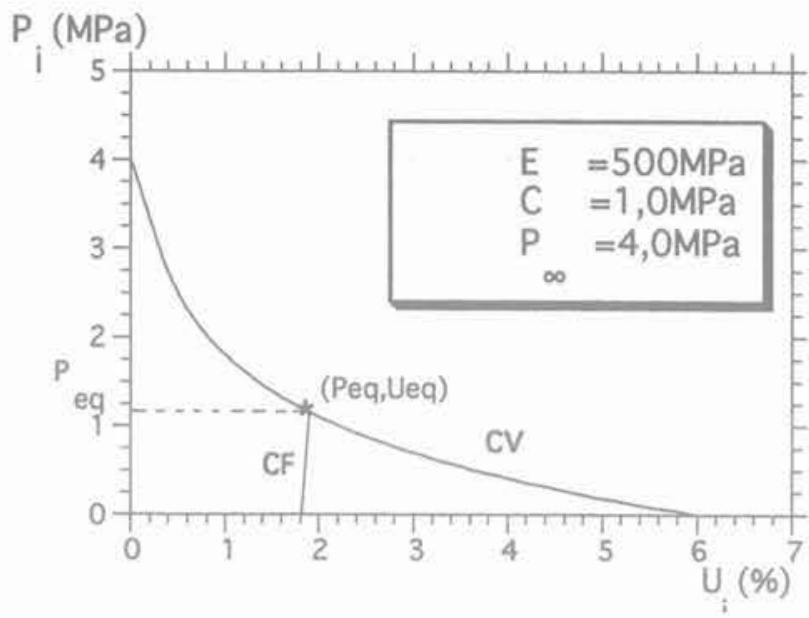

FG.8 Équilibre du tunnel dans le diagramme $\mathrm{P}_{1} \times \mathrm{U}_{\mathrm{l}}$ pour l'exemple étudié. Tunnel equilibrium in a diagram $\mathrm{P} \times \mathrm{U}_{\mathrm{p}}$ for the studied case.

En élasticité, nous avons montré que la différence

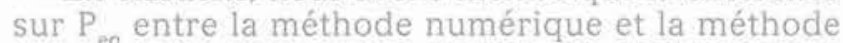
approchée reste importante, jusqu'à $20 \%$ pour les soutènements raides. L'erreur est toujours de même signe: la méthode convergence-confinement sous-estime la pression à l'équilibre, ce qui ne va pas dans le sens de la sécurité de l'ouvrage.

En résumé, la méthode approchée «convergenceconfinement ») peut conduire à des imprécisions importantes, surtout dans le cas des soutènements de raideur forte ou moyenne, posés près du front; ces imprécisions viennent de l'estimation de la convergence $\mathrm{U}_{0}$ avant pose: la valeur de $U_{0}$ ne dépend pas seulement de la loi rhéologique du massif et de la distance $d_{0}$ de pose au front, comme le prévoit la méthode convergence-confinement, mais elle dépend également de la rigidité du soutènement.

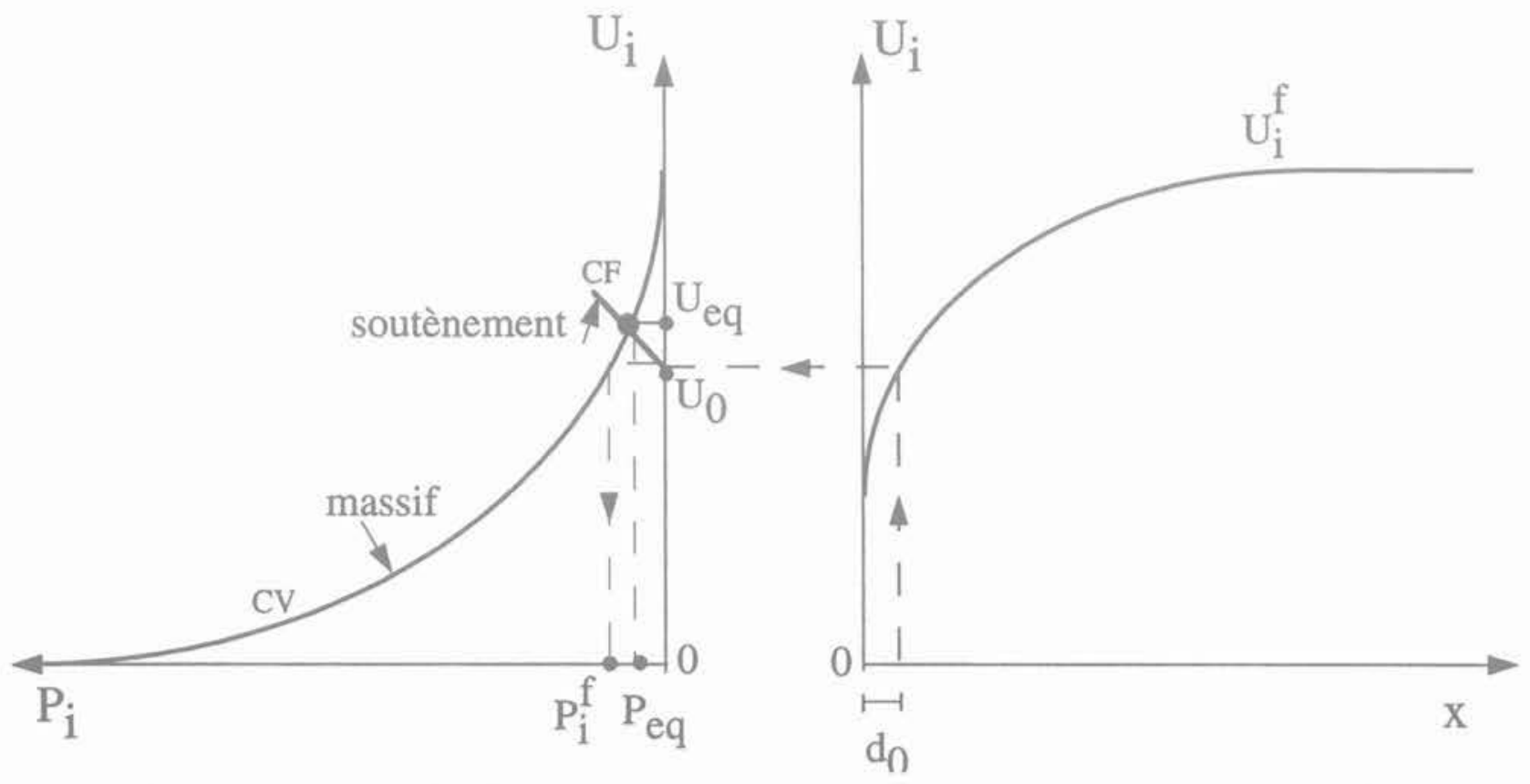

FiG.9 Application de la méthode convergence-confinement. Calculation with the convergence-confinement method. 


\section{Nouvelle méthode simplifiée de calcul des tunnels en élasticité et plasticité: "la nouvelle méthode implicite "}

La «Nouvelle Méthode Implicite » pour l'étude du dimensionnement des tunnels a été proposée initialement dans l'article de Bernaud et Rousset (1992). Une application de cette méthode à un cas particulier d'un tunnel creusé dans une argile profonde du bassin parisien est donnée dans Bernaud et Rousset (1994).

Il s'agit d'une méthode fondée sur les principes de base de la méthode convergence-confinement, qui permet de tenir compte de façon correcte du caractère couplé du problème d'interaction massif-soutènement, par l'intermédiaire de la convergence $\mathrm{U}_{0}$ de la paroi au moment où est posé le soutènement. En particulier, «La Nouvelle Méthode Implicite » tient compte de la dépendance de $U_{0}$ par rapport à la raideur du soutènement.

Par la suite, on rappelle les principes de base de cette nouvelle méthode et on expose les derniers développements réalisés.

\section{1}

\section{Principes de base de la nouvelle méthode}

Fondamentalement, la nouvelle méthode implicite consiste à déduire la courbe $\mathrm{U}(\mathrm{x})$ donnant la convergence du tunnel soutenu en fonction de la distance $x$ au front, de la courbe de convergence du tunnel non soutenu $\mathrm{U}^{f}(\mathrm{x})$ par une transformation géométrique simple.

Pour ce qui concerne le tunnel non soutenu, plusieurs auteurs (Panet et Guénot, 1982; Corbetta, 1991; Bernaud et Rousset, 1992) ont proposé une forme approchée de la courbe de convergence $\mathrm{U}^{\mathrm{f}}(\mathrm{x})$ (en élasticité et plasticité) qui donne, en général, une bonne concordance avec les calculs numériques axisymétriques.

Par la suite, on introduit la fonction de forme $\mathrm{a}^{0}(\mathrm{x})$ (fonction croissante entre 0 et 1) du tunnel non soutenu définie par:

$$
a^{0}(x)=\frac{U_{i}^{f}(x)-U_{i}^{p}(0)}{U_{i}^{f}(\infty)-U_{i}^{f}(0)}
$$

où $\mathrm{U}_{f}=\mathrm{U}_{i}^{f}(0)$ est la convergence au front et $\mathrm{U}_{-}=$ $\mathrm{U}_{i}(\infty)$ la convergence du tunnel non soutenu loin du front de taille.

Il est remarquable de constater que, dans tous les cas traités ici, l'expression analytique suivante donne une très bonne approximation du profil de convergence du tunnel non soutenu:

$$
a^{0}(x)=1-\left(\frac{0,84 R_{p}}{x+0,84 R_{p}}\right)^{2}
$$

et pour la convergence au front:

$$
\mathrm{U}_{i}^{f}(0)=0,29 \mathrm{U}_{i}^{f}(\infty)
$$

$\left(\mathrm{R}_{p}\right.$ est le rayon plastique du tunnel non soutenu à l'équilibre; en élasticité $R_{p}=R_{i}$ ).

Néanmoins, les principes de base de la NMI ainsi que son application ne dépendent pas de ce choix. Le lecteur intéressé par cette méthode pourra proposer une expression $\mathrm{a}^{\circ}(\mathrm{x})$ à sa convenance.

Pour le tunnel soutenu, les calculs numériques 3D axisymétriques montrent que la forme de la courbe de convergence en fonction de la distance au front $\mathrm{U}(\mathrm{x})$ dépend de la raideur du soutènement. En effet, ces calculs montrent que bien que $U_{i}$ soit toujours une fonction croissante de $x$ entre $U_{f}$ pour $x=0$ et $U_{\text {eq }}$ pour $\mathrm{X}=\infty$, la variation de $\mathrm{U}$ en fonction de $\mathrm{x}$ entre le front et le soutènement est beaucoup plus forte lorsque la raideur du soutènement $\mathrm{K}_{5}$ augmente.

La méthode classique convergence-confinement ne tient pas compte de cette dépendance.

Pour tenir compte de ce phénomène, une voíe à explorer, suggérée par les formes des différentes courbes $\mathrm{a}(\mathrm{x})=\frac{\mathrm{U}_{\mathrm{i}}(\mathrm{x})-\mathrm{U}_{\mathrm{i}}(0)}{\mathrm{U}_{\mathrm{i}}(\infty)-\mathrm{U}_{\mathrm{i}}(0)}$ consiste à substituer à la fonction $x \rightarrow a^{0}(x)$ (du tunnel non soutenu) la fonction $\mathrm{a}^{\mathrm{s}}(\mathrm{x})$ construite à partir de celle-ci par simple affinité d'axe vertical, le rapport d'affinité $\alpha$ ne dépendant que de la raideur réduite du soutènement $\mathrm{K}_{\mathrm{s}}^{\prime}$ pour une loi de comportement donnée:

$$
\mathrm{a}^{\mathrm{s}}(\mathrm{x})=\mathrm{a}^{0}(\alpha \mathrm{x}) \text { avec } \alpha=\alpha\left(\mathrm{K}_{\mathrm{s}}^{\prime}\right)
$$

Enfin, la solution cherchée $\left(\mathrm{P}_{\mathrm{eq}}, \mathrm{U}_{e \mathrm{q}}\right)$ est l'intersection des deux courbes de convergence CV et de confinement CF:

$$
\left\{\begin{array}{l}
\mathrm{P}_{\mathrm{eq}}=\mathrm{CV}\left(\mathrm{U}_{\mathrm{eq}}\right) \\
\mathrm{P}_{\mathrm{eq}}=\mathrm{CF}\left(\mathrm{U}_{\mathrm{eq}}-\mathrm{U}_{0}\right)
\end{array}\right.
$$

Dans le système (8), la valeur de $\mathrm{U}_{0}$ dépend implicitement de la solution $\mathrm{U}_{\mathrm{eq}}$ par:

$$
\mathrm{U}_{0}=\mathrm{a}^{\mathrm{s}}\left(\mathrm{d}_{0}\right)\left(\mathrm{U}_{\mathrm{eq}}-\mathrm{U}_{\mathrm{f}}\right)+\mathrm{U}_{\mathrm{f}}
$$

\section{2}

\section{Développement de la nouvelle méthode}

La nouvelle méthode nécessite l'évaluation de la fonction de soutènement $\alpha$ et de la convergence au front $\mathrm{U}_{f}$ pour chaque loi de comportement du massif choisie. Pour déterminer ces deux fonctions, nous avons donc réalisé une série de calculs numériques, à l'aide de la méthode d'activation/désactivation, dans des milieux élastiques et élastoplastiques incompressibles. Le calage a été réalisé sur les paramètres indépendants suivants:

$$
P_{\infty}^{\prime}=0,008 ; d_{0}^{\prime}=2 / 3 ; N_{s}=\frac{P_{\infty}}{C}=1,0 ; 2,0 ; 3,0 ; 4,0 ; 5,0
$$

et $\mathrm{K}_{\mathrm{s}}^{\prime}=0,072 \rightarrow 72$.

La figure 10 montre la fonction $\alpha$ en fonction de $\mathrm{K}^{\prime}$ obtenue par les calculs numériques en élasticité et plasticité (critère de Tresca) avec: 


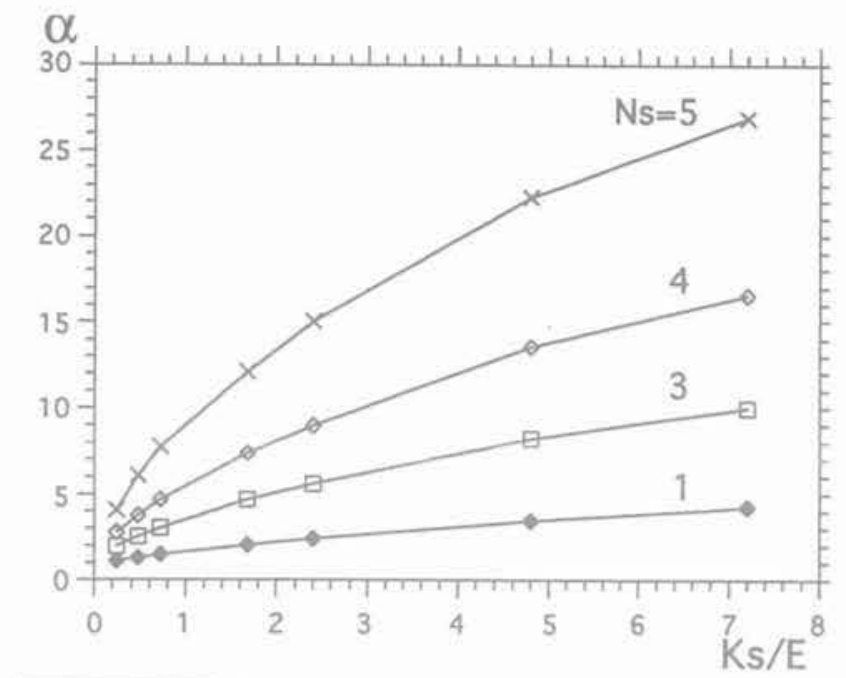

FIG. 10 Fonction de soutènement $\alpha$ pour plusieurs $\mathrm{N}_{5^{*}}$.

Lining function $\alpha$ for many Ns.

$P_{s}^{\prime}=0,008 ; d_{0}^{\prime}=2 / 3 ; N_{s}=\frac{P_{s}}{C}=1,0 ; 3,0 ; 4,0 ; 5,0$

et $\mathrm{K}_{\mathrm{s}}^{\prime}=0,072 \rightarrow 72$.

Sur la figure 11, nous avons tracé la fonction réduite de soutènement $\alpha^{*}=\alpha R / R_{n}$ correspondant à chaque $\alpha$. Il est intéressant d'observer sur la figure 11 que les fonctions $\alpha^{*}$ correspondant aux différentes valeurs de $\mathrm{N}_{\mathrm{s}}$ sont très similaires $\left(\mathrm{N}_{\mathrm{s}}=\right.$ coefficient de stabilité, eq.16).

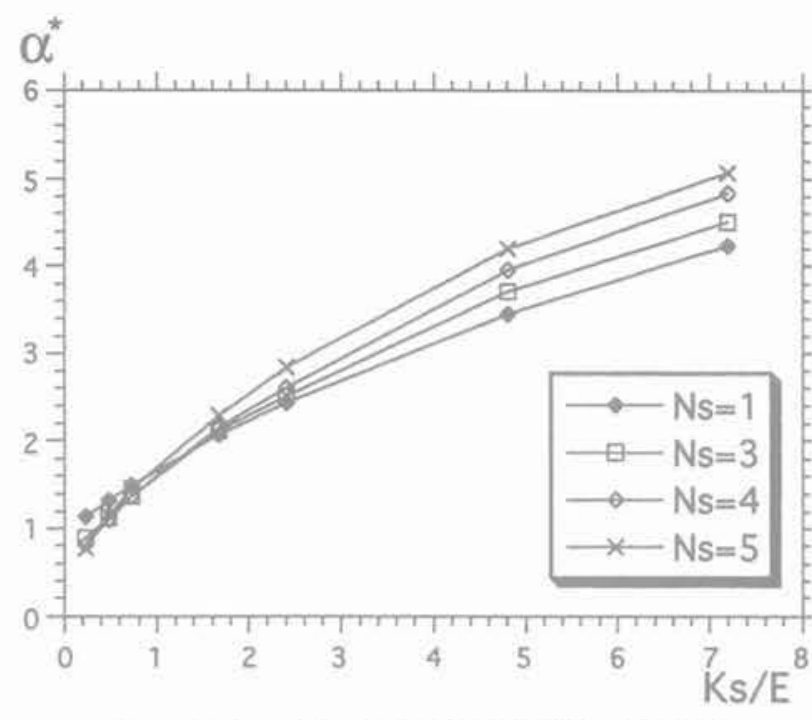

FiG. 11 Fonction réduite de soutènement $\alpha^{*}$. Non dimensional lining function $\alpha^{*}$.

Dans l'esprit de construire une méthode approchée simple, on propose ici de choisir une fonction $\alpha^{*}=\alpha$ (Fig. 12) unique, capable de représenter l'ensemble dü comportement élastique et élastoplasique (Tresca, Mises). On donne:

$$
\alpha_{\text {moy }}^{*}=1,82 \sqrt{\mathrm{K}_{\mathrm{s}}^{\prime}} \text { valable pour } \mathrm{K}_{\mathrm{s}}^{\prime} \geq \frac{\mathrm{R}_{\mathrm{i}}^{2}}{\left(1,82 \mathrm{R}_{\mathrm{p}}\right)^{2}}
$$

A posteriori on vérifie que cette fonction $\alpha$ choisie conduit à de bons résultats lorsqu'on fait varier l'ensemble des paramètres indépendants du problème.

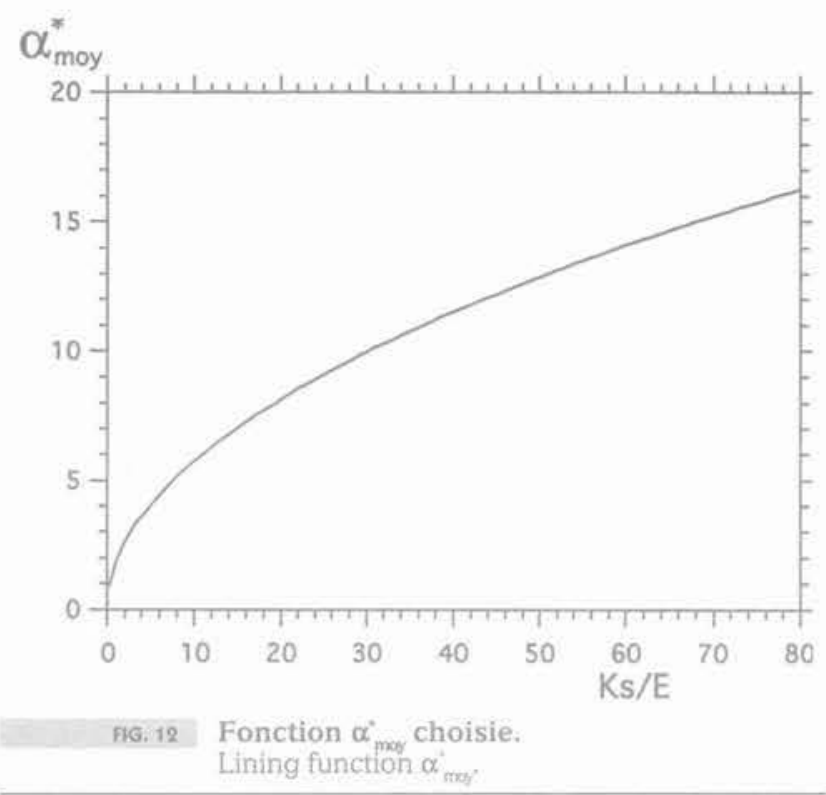

D'autres lois de comportement plastique ont été étudiées, notamment le cas d'un matériau de Tresca avec écrouissage positif.

Dans ce qui suit on explicite les fonctions de soutènement pour les matériaux de Coulomb et Hoek-Brown (Hoek et Brown, 1988; Hoek et al., 1992) sans dilatance.

$$
\begin{aligned}
& \text { • Coulomb }\left(F=K_{p} \sigma_{1}-\sigma_{3}-2 C \sqrt{K_{p}}\right. \text { avec } \\
& \left.\sigma_{1}>\sigma_{2}>\sigma_{3} \text { contraintes principales }\right) \\
& \alpha^{*}=\alpha_{\text {moy }}^{*}+0,035 \phi
\end{aligned}
$$

$\phi$ est l'angle de frottement en degrés et $K_{p}=(1+\sin \phi) /(1-\sin \phi)$.

- Hoek - Brown

$$
\begin{aligned}
& \left(\mathrm{F}=\sigma_{1}-\sigma_{3}-\sqrt{\mathrm{s} \sigma_{\mathrm{c}}^{2}-\mathrm{m} \sigma_{1} \sigma_{\mathrm{c}}} \text { avec } \mathrm{s}=1\right) \\
& \alpha^{*}=\alpha_{\text {moy }}^{*}+0,15 \mathrm{~m}
\end{aligned}
$$

- Hoek - Brown Modifié

$$
\begin{aligned}
& \left(F=\sigma_{1}-\sigma_{3}-\sigma_{c}\left(-m_{b} \frac{\sigma_{1}}{\sigma_{c}}\right)^{a}\right) \\
& \alpha^{*}=\alpha_{\text {moy }}^{*}+0,25 m_{b}
\end{aligned}
$$

avec $a, m$ et $m_{b}$ des constantes du matériau et $\sigma_{c}$ la limite en compression simple.

Les équations (11) à (13) sont valables pour $\alpha^{*} \geq$ $\mathrm{R} / \mathrm{R}_{\mathrm{p}}$, sinon on utilise $\alpha^{*}=\mathrm{R}_{\mathrm{i}} / \mathrm{R}_{\mathrm{p}}$ qui correspond au tunnel non soutenu.

Il est intéressant de constater que toutes les fonctions $\alpha^{*}$ ont été déduites, de façon très simple à partir de la fonction $\alpha_{\text {moy }}^{*}$ (valable pour l'élasticité et Tresca, Mises).

Finalement, pour compléter le développement de la nouvelle méthode, la convergence au front de taille doit être calculée.

En élasticité, on constate que la valeur de $\mathrm{U}_{f}$ ne dépend presque pas de la rigidité du soutènement et on donne:

$$
\mathrm{U}_{f}=0,27 \mathrm{U}_{\infty} \text { avec } \mathrm{U}_{\infty}=\frac{1+\mathrm{v}}{\mathrm{E}} \mathrm{P}_{\infty}
$$


En plasticité l'influence de la rigidité du soutènement sur la valeur de $U_{\text {f }}$ est plus importante. Néanmoins, ce phénomène est restreint aux faibles valeurs de $\mathrm{K}_{\mathrm{s}}^{\prime}$, comme le montre la figure 13 .

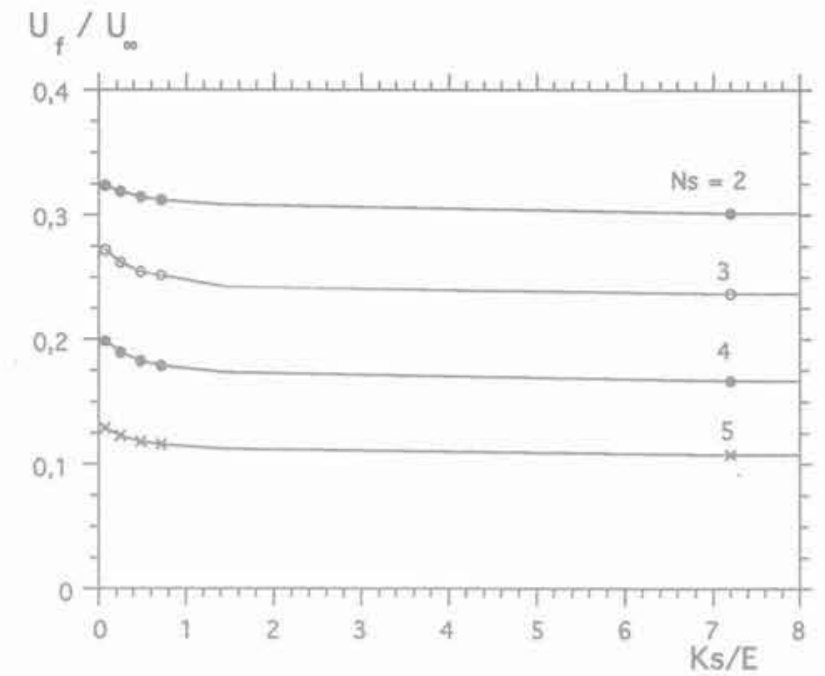

FiG. $13 \mathrm{U}_{\mathrm{t}} / \mathrm{U}_{-}$en fonction de $\mathrm{K}_{\mathrm{s}}^{\prime}$, pour plusieurs $\mathrm{N}_{s}$ (Tresca, $d_{0}=2 / 3 R_{0}$ ).

$\mathrm{U}, \mathrm{U}$.

Par la suite on propose une valeur moyenne de $\mathrm{U}_{f}$ pour Tresca, Coulomb et Hoek-Brown:

$$
\frac{U_{f}}{U_{s}}=0,413-0,0627 N_{s} \quad 1<N_{s} \leq 5
$$

Pour Tresca $(\phi=0)$ et Coulomb

$$
N_{s}=\frac{2 P_{\infty}}{R_{c}} \text { et } R_{c}=\frac{2 C \cos \phi}{1-\sin \phi}
$$

Pour Hoek-Brown, $N_{s}=\frac{2 P_{\alpha}}{\sqrt{\sigma_{c}^{2}-m \sigma_{c} \sigma_{y}}}$

$\sigma_{y}=\frac{-m \sigma_{c}}{8}-P_{\infty}+\frac{\sigma_{c}}{2} \sqrt{\frac{m^{2}}{16}+\frac{m P_{s c}}{\sigma_{c}}+1}$

Pour Hoek-Brown Modifié l'équation (15) n'est pas valable et on propose:

$$
\begin{gathered}
\frac{U_{f}}{U_{\infty}}=(0,75-0,5 \mathrm{a})-0,133 \mathrm{~N}_{\mathrm{s}} \\
\mathrm{N}_{\mathrm{s}}=\frac{2 \mathrm{P}_{\mathrm{ec}}}{\sigma_{\mathrm{c}}\left(-\frac{\mathrm{m}_{\mathrm{b}} \sigma_{y}}{\sigma_{\mathrm{c}}}\right)^{\mathrm{a}}}
\end{gathered}
$$

Où $\sigma_{\mathrm{y}}$ est obtenu par:

$$
\frac{\sigma_{c}}{2}\left(-\mathrm{m}_{b} \frac{\sigma_{y}}{\sigma_{c}}\right)^{\mathrm{a}}-\sigma_{y}-\mathrm{P}_{\infty}=0
$$

La valeur exacte de $\mathrm{U}_{\infty}$ pour les diverses lois de comportement étudiées ici est donnée dans l'annexe 1.

Finalement à partir des données ci-dessus, la solution du problème du tunnel soutenu est facilement obtenue par la résolution du système (8), que l'on explicite dans l'annexe 1.

\section{Application de la Nouvelle Méthode Implicite}

La nouvelle méthode a été validée sur une très large gamme des paramètres indépendants de chaque loi de comportement étudiée.

Sur la figure 14 on illustre la différence entre les résultats donnés par la méthode classique convergenceconfinement et ceux donnés par la nouvelle méthode implicite, dans un cas particulier (Tresca $\mathrm{N}_{\mathrm{s}}=3 ; \mathrm{K}_{\mathrm{s}}^{\prime}=7,2$; $\left.d_{0}^{\prime}=2 / 3 ; P_{\infty}^{\prime}=0,008\right)$

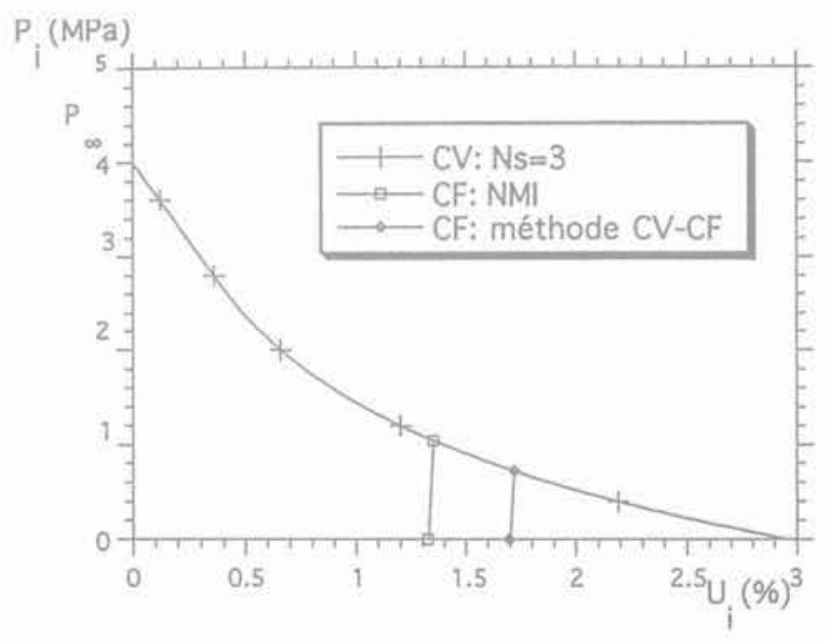

Fic. 14 Comparaison entre la méthode CV-CF et la NMI.

Comparison between CV-CF method and NIM method.

On constate que dans le cas de la figure 14, l'erreur commise par la méthode CV-CF sur la pression à l'équilibre est de $-30 \%$. Par ailleurs, on peut clairement observer sur la figure14 que cette erreur vient de la mauvaise estimation de la convergence $\mathrm{U}_{0}$ qui conditionne la position de la courbe de confinement sur le diagramme $\mathrm{P}_{\mathrm{i}}-\mathrm{U}_{\mathrm{i}}$.

Finalement, la «Nouvelle Méthode Implicite» a été testée sur un nombre plus vaste de cas, pour diverses lois de comportement. Le tableau 1 illustre quelques résultats de validation. L'erreur entre la nouvelle méthode et le calcul numérique reste très satisfaisante pour une étude géotechnique. En valeur absolue, elle est inférieure à $10 \%$ en moyenne sur les paramètres dimensionnants du tunnel $\mathrm{P}_{\text {eq }}$ et $\mathrm{U}_{\text {eq. }}$.

Un autre intérêt des méthodes simplifiées de calcul des tunnels est de déterminer la convergence à l'instant de pose du soutènement et donc, la pression $\mathrm{P}_{0}$ qu'il faut appliquer à la paroi du tunnel pour obtenir cette convergence $\mathrm{U}_{0}$, pour ensuite réaliser des calculs de tunnels en déformations planes avec des géométries et des chargements plus complexes. Le plus souvent, on remplace la pression $\mathrm{P}_{0}$ par le taux de déconfinement $\lambda_{0}\left(\lambda_{0}=1-\mathrm{P}_{\alpha} / \mathrm{P}_{\infty}\right)$ au moment de la pose du soutènement, comme le montre le schéma de la figure 15.

Par la suite on s'intéresse aux tunnels creusés dans les milieux viscoplastiques et on propose l'extension de la “Nouvelle Méthode Implicite» aux tunnels excavés à vitesse constante dans de tels milieux. 
TABLEAUI Validation de la NMI pour diverses lois de comportement.

$\left(\mathrm{P}_{\mathrm{eq}}^{\mathrm{c}}\right.$ : calcule EF; $\mathrm{P}_{\mathrm{eq}}^{\mathrm{i}}$ : calcul $\left.\mathrm{NMI}\right)$

Validity of the NIM for many constitutives laws.

$\left(\mathrm{P}_{\mathrm{eq}}^{\prime c} ;\right.$ FEM calculation; $\mathrm{P}_{\mathrm{eq}}^{\prime \prime \prime} ;$ NMI calculation),

\begin{tabular}{|c|c|c|c|c|c|c|}
\hline Lois de comportement & $P_{-}^{\prime}$ & $\mathrm{d}_{0}$ & $K_{s}^{\prime}$ & $\begin{array}{l}P_{s q}^{c} \\
\times 10^{3}\end{array}$ & $\begin{array}{l}P_{e q}^{P^{3}} \\
\times 10^{3}\end{array}$ & $\frac{\mathrm{P}_{\mathrm{eq}}^{\mathrm{C}} \mathrm{P}_{\mathrm{eq}}^{\mathrm{iE}}}{\mathrm{P}_{\mathrm{eq}}^{\mathrm{c}}}$ \\
\hline $\begin{array}{l}\text { TRESCA } \\
C=1,0 \mathrm{MPa} \\
\mathrm{C}=1,33 \mathrm{MPa}\end{array}$ & $\begin{array}{l}0,008 \\
0,008\end{array}$ & $\begin{array}{l}1 / 3 \\
1,0\end{array}$ & $\begin{array}{r}7,2 \\
72,0\end{array}$ & $\begin{array}{l}2,96 \\
18\end{array}$ & $\begin{array}{l}3,0 \\
17\end{array}$ & $\begin{array}{c}-2 \\
6\end{array}$ \\
\hline $\begin{array}{l}\text { COULOMB } \\
\mathrm{C}=0,8 \mathrm{MPa} \quad \emptyset=4^{\circ} \\
\mathrm{C}=0,8 \mathrm{MPa} \emptyset=15^{\circ} \\
\mathrm{C}=0,8 \mathrm{MPa} \quad \emptyset=30^{\circ}\end{array}$ & $\begin{array}{l}0,008 \\
0,008 \\
0,01\end{array}$ & $\begin{array}{l}2 / 3 \\
5 / 3 \\
5 / 3\end{array}$ & $\begin{array}{l}0,24 \\
2,40 \\
7,20\end{array}$ & $\begin{array}{l}1,52 \\
0,70 \\
0,618\end{array}$ & $\begin{array}{l}1,50 \\
0,62 \\
0,62\end{array}$ & $\begin{array}{c}1 \\
10 \\
0\end{array}$ \\
\hline $\begin{array}{l}\text { HOEK-BROWN } \\
\sigma_{c}=1,5 \mathrm{MPa} m=1 \\
\sigma_{c}=1,5 \mathrm{MPa} m=10 \\
\sigma_{c}=5,0 \mathrm{MPa} m=1,0 \\
\sigma_{c}=5,0 \mathrm{MPa} m=1,0\end{array}$ & $\begin{array}{l}0,008 \\
0,008 \\
0,008 \\
0,008\end{array}$ & $\begin{array}{l}23 \\
23 \\
2 / 3 \\
2 \beta\end{array}$ & $\begin{array}{l}0,72 \\
0,72 \\
0,72 \\
72,0\end{array}$ & $\begin{array}{l}1,62 \\
0,52 \\
0,94 \\
2,20\end{array}$ & $\begin{array}{l}1,48 \\
0,54 \\
0,90 \\
1,94\end{array}$ & $\begin{array}{c}8 \\
-2 \\
5 \\
11\end{array}$ \\
\hline $\begin{array}{l}\text { HOEK-BROWN MODIFIE } \\
\sigma_{c}=2,5 \mathrm{MPa} \mathrm{m}_{\mathrm{b}}=1,0 \mathrm{a}=0,3 \\
\sigma_{\mathrm{c}}=3,5 \mathrm{MPa} \mathrm{m}_{\mathrm{b}}=10 \mathrm{a}=0,3 \\
\sigma_{\mathrm{c}}=2,5 \mathrm{MPa} \mathrm{m}_{\mathrm{b}}=2,0 \mathrm{a}=0,5 \\
\sigma_{\mathrm{c}}=2,5 \mathrm{MPa} \mathrm{m}_{\mathrm{b}}=1,5 \mathrm{a}=0,4\end{array}$ & $\begin{array}{l}0,008 \\
0,008 \\
0,008 \\
0,008\end{array}$ & $\begin{array}{l}2 / 3 \\
23 \\
2 / 3 \\
2 / 3\end{array}$ & $\begin{array}{l}0,48 \\
0,48 \\
7,2 \\
7,2\end{array}$ & $\begin{array}{l}1,30 \\
0,24 \\
1,18 \\
1,28\end{array}$ & $\begin{array}{l}1,24 \\
0,24 \\
1,22 \\
1,38\end{array}$ & $\begin{array}{l}6 \\
0 \\
-3 \\
-7\end{array}$ \\
\hline
\end{tabular}

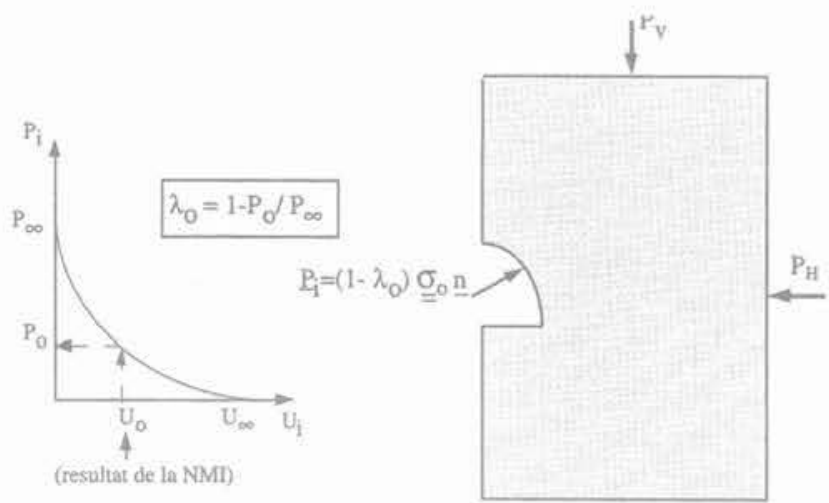

FG. 15 Applícation du taux de déconfinement à un calcul en déformation plane. Application of the ration $\lambda_{\rho}$ in a plane strain model.
5

\section{Extension de la nouvelle méthode implicite aux massifs viscoplastiques}

Dans cette partie, on présente l'extension de la nouvelle méthode implicite au cas du massif élastoviscoplastique incompressible. Une dimension nouvelle intervient dans le problème: le temps. Dans le cas des tunnels creusés dans de tels milieux, l'effet du temps est dû à deux principaux facteurs: la viscosité de la roche (comportement rhéologique) et l'histoire de creusement de l'ouvrage (notamment la vitesse de creusement). On étudie le cas du tunnel circulaire avec vitesse d'avancement du front de taille constante (problème axisymétrique stationnaire), de façon à minimiser le nombre de variables du problème et à rendre possible l'étude paramétrique.

\section{1}

\section{Lois de comportement viscoplastique et paramètres indépendants}

Dans le cadre de cette étude, le massif choisi suit un comportement mécanique élastoviscoplastique avec une loi de comportement de Bingham. Les évolutions sont considérées isothermes, on choisit comme loi d'évolution viscoplastique la forme suivante (Perzyna, 1966, Zienkiewicz et Cormeau, 1974):

$$
\dot{\underline{\varepsilon}}^{\mathrm{vp}}=\frac{1}{\eta}<\frac{\mathrm{F}(\underline{\underline{\underline{\sigma}}})}{\mathrm{F}_{0}}>^{n} \cdot \frac{\partial \mathrm{G}(\underline{\underline{\underline{\sigma}}})}{\partial \underline{\underline{\sigma}}}
$$

où $F(\underline{\underline{\sigma}})$ : critère de plasticité

$\mathrm{G}(\underline{\underline{\sigma}})$; potentiel plastique

gvp : tenseur de vitesse de déformation viscoplastique

$\mathrm{F}_{0} \quad$ : contrainte de référence

$\eta, \mathrm{n}$ : constantes de viscosité

Dans cette étude on se restreint au cas d'un exposant $n=1$, avec un critère et potentiel de Tresca.

Le tenseur de vitesse de déformation total s'écrit de façon classique:

$$
\underline{\underline{\dot{\varepsilon}}}=\underline{\underline{\varepsilon}}^{\mathrm{e}}+\underline{\underline{\varepsilon}}^{\mathrm{vp}} \text { avec } \underline{\underline{\varepsilon}}^{\mathrm{e}}=\frac{(1+v)}{\mathrm{E}} \dot{\underline{\sigma}}-\frac{v}{E}(\operatorname{tr} \underline{\underline{\sigma}}) \underline{\underline{1}}
$$

Pour ce problème, on peut montrer (Bernaud, 1991) qu'il y a cinq paramètres adimensionnels indépendants: - deux caractérisant le massif $\left(\mathrm{E}^{*}=\mathrm{E} / \mathrm{C}\right.$ et $\left.\mathrm{N}_{\mathrm{s}}=\mathrm{P}_{\infty} / \mathrm{C}\right)$; - un caractérisant le soutènement $\left(\mathrm{K}_{\mathrm{s}}{ }^{*}=\mathrm{K}_{\mathrm{s}} / \mathrm{C}\right.$ ou $\left.\mathrm{K}_{\mathrm{s}}^{\prime}=\mathrm{K}^{*} / \mathrm{E}^{*}\right)$;

- deux caractérisant le chargement $\left(\mathrm{d}_{0}^{\prime}=\mathrm{d}_{0} / \mathrm{R}_{1}\right.$ et $V^{*}=(\eta V) /(R C)$, la vitesse réduite $)$. 


\section{Calcul numérique en viscoplasticité}

Afin d'illustrer le problème des tunnels creusés dans les milieux viscoplastiques on donne ci-dessous les résultats de deux calculs numériques (méthode d'activation/désactivation) avec une même loi de comportement de Tresca $\left(\mathrm{N}_{\mathrm{s}}=3\right)$ pour les paramètres suivants: $d_{0}^{\prime}=1 ; V^{*}=150 ; K_{s}^{s}=0,72$ et $V^{*}=500 ; K_{s}^{\prime}=0,24$.

Sur les figures 16 et 17 , on peut observer clairement le caractère couplé du problème, mis en évidence dans ces résultats par la vitesse de creusement réduite $V^{*}$ et par la rigidité du soutènement $\mathrm{K}_{\text {. }}$. Si le milieu était élastoplastique, la convergence $\mathrm{U}_{0}$ plus faible (et donc $\mathrm{U}_{\mathrm{p}}$ ) serait celle correspondant à la rigidité la plus élevée (puisque tous les autres paramètres du problème sont égaux), ce qui n'est pas le cas ici dans ces deux exemples. Ceci illustre bien que dans les milieux viscoplastiques, la vitesse de creusement est un paramètre fondamental qui conditionne l'équilibre de l'ouvrage.

On constate bien que, comme prévu, l'équilibre du tunnel pour chaque cas étudié se situe sur la courbe de convergence à long terme (plasticité) du massif.

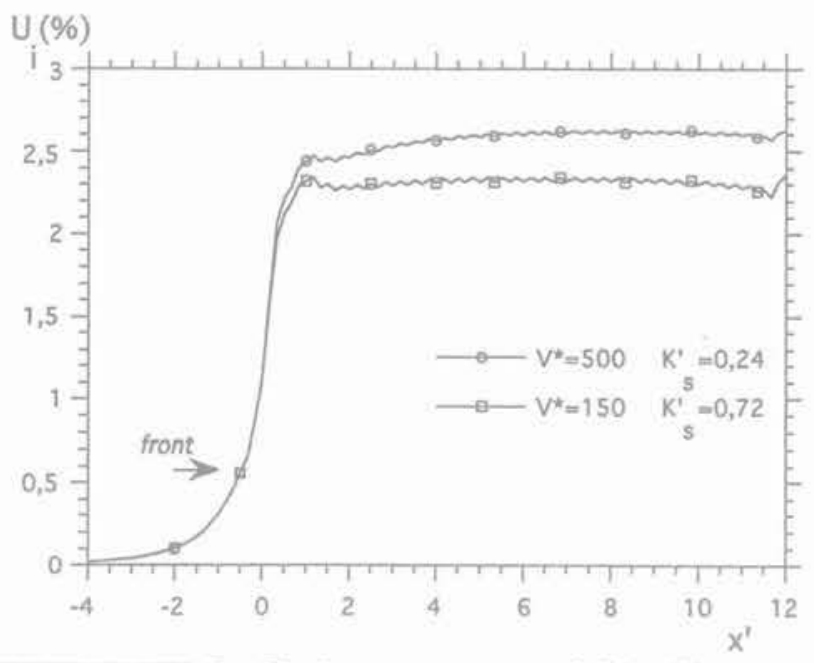

FIG.16e Profils des convergences à l'équilibre. Convergence versus $x$ ' at equilibrium.

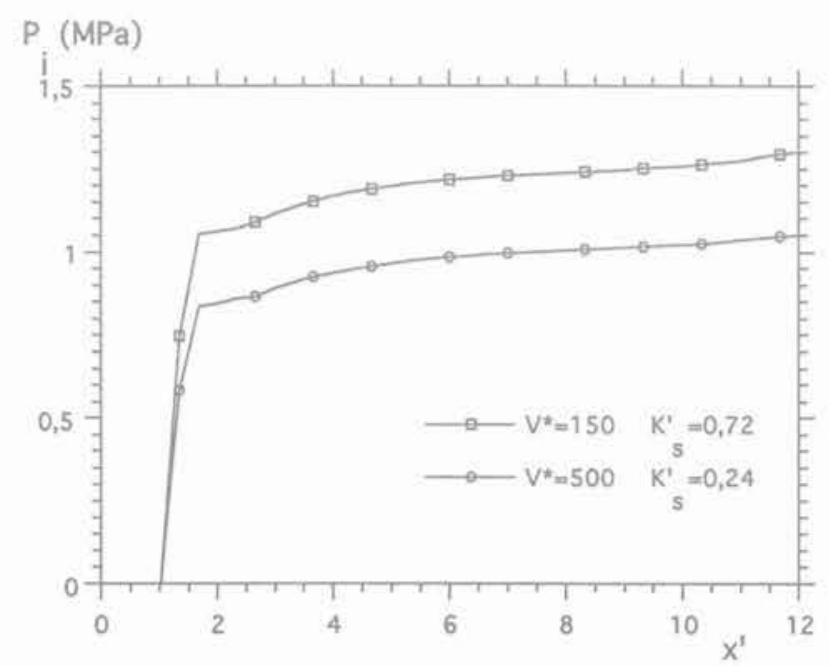

FlG. 166 Profils des pressions à l'équilibre. Lining pressure versus $x$ at equilibrium.
$P_{1}(\mathrm{MPa})$

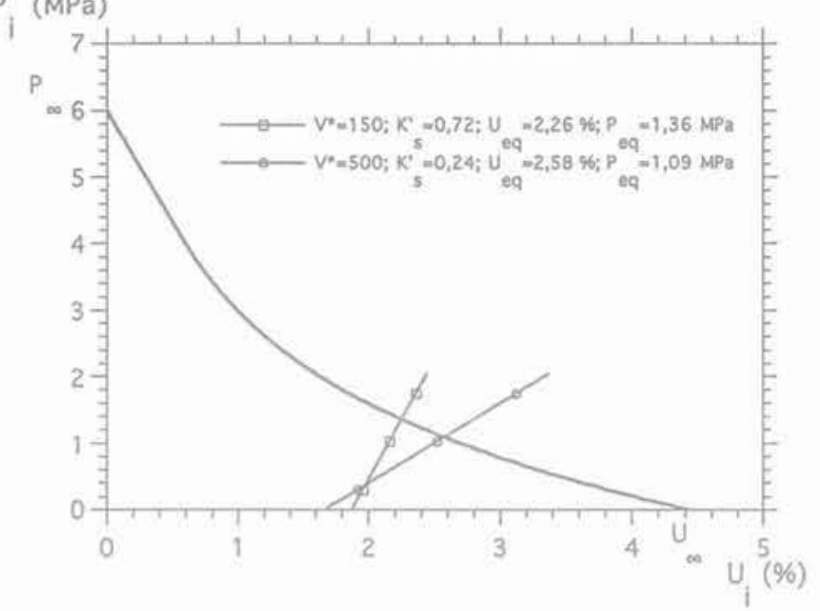

FiG.17 Équilibre du tunnel pour les deux cas traités.

Equilibrium of the tunnel for the two cases.

\section{3}

\section{Développement de la nouvelle méthode pour les milieux viscoplastiques}

Le développement de la Nouvelle Méthode Implicite au matériau viscoplastique suit la même démarche que celle adoptée précédemment pour les milieux élastique et élastoplastique. Ainsi, on doit initialement chercher une fonction de forme du tunnel non soutenu.

\section{S.3.1}

\section{Tunnel non soutenu}

Afin d'illustrer l'influence de la vitesse de creusement dans les milieux viscoplastiques, nous avons tracé sur la figure 18 , les courbes de convergence en fonction de la distance au front obtenues numériquement pour plusieurs vitesses de creusement, dans le cas suivant: $\mathrm{N}_{5}=2, \mathrm{E}^{*}=250$.

La vitesse $\mathrm{V}$ étant considérée constante pour une étude donnée, $\mathrm{x}$ et $\mathrm{t}$ sont donc reliés par la relation simple: $\mathrm{x}=\mathrm{Vt}$. Ainsi pour une vitesse $\mathrm{V}$ non nulle et

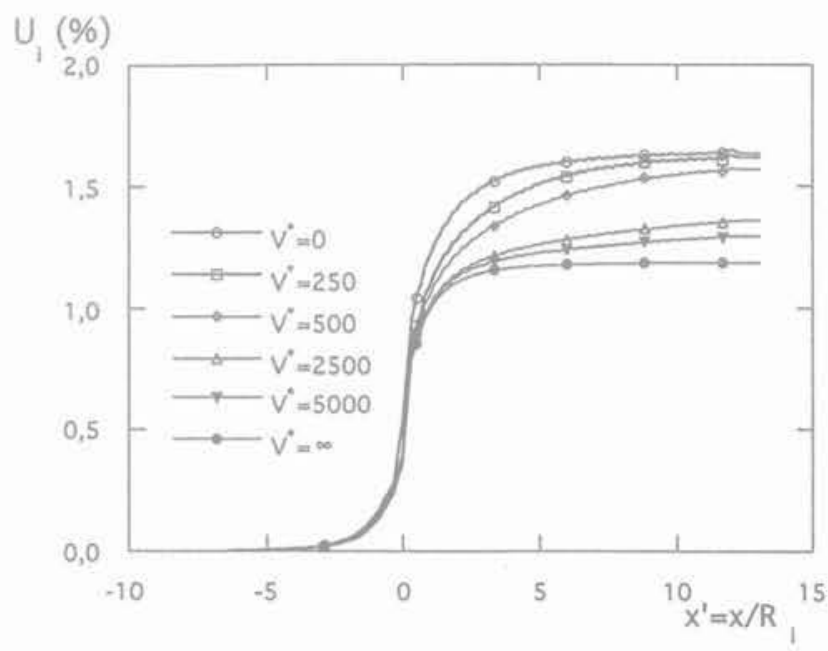

FIG. 18 Convergence en fonction de la distance au front de taille pour plusieurs vitesses. Convergence versus the distance from the tunnel face for many tunnel advance rates. 
non infinie, chacune des courbes de convergence de la figure 18 représente:

- pour un instant donné, la convergence de la paroi du tunnel en fonction de la distance au front;

- pour un point donné, d'abscisse $x$ et d'une vitesse de creusement V, l'évolution en fonction du temps de la convergence en ce point. L'abscisse est alors le temps: mais l'échelle dépend de la vitesse V.

On peut observer sur ces courbes que plus $V$ est grande, plus il faudra de temps pour que la convergence à la paroi se stabilise. Dans le cas du tunnel non soutenu, à la stabilisation finale $\left(\hat{\varepsilon}^{\mathrm{vp}}=0 ; F=0\right.$ en tout point du massif) toutes les courbes correspondant à différentes vitesses retrouveront la courbe de convergence du tunnel en plasticité (soit $\mathrm{V}=0$ ).

Remarques:

$\mathrm{V}=0$ correspond à un calcul plastique.

$\mathrm{V}=\infty$ correspond à un calcul élastique.

$\forall \mathrm{V} \mathrm{U}^{\prime}(\mathrm{x}=\infty)=\mathrm{U}$ convergence du tunnel non soutenu en plasticité.

En suivant la même démarche de l'élasticité et plasticité, on définit la fonction de forme $\mathrm{a}_{\mathrm{vp}}^{0}(\mathrm{x})$ du tunnel non soutenu viscoplastique par:

$$
a_{v p}^{0}(x)=\frac{U_{i}^{p}(x)-U_{i}^{f}(0)}{U_{\infty}-U_{i}^{f}(0)}
$$

Sur la figure 19 , nous avons tracé les fonctions de forme $\mathrm{a}_{\mathrm{vp}}^{0}(\mathrm{x})$ du tunnel non soutenu correspondant aux calculs numériques donnés précédemment sur la figure 18.

Dans le cas d'un calcul viscoplastique, l'allure des courbes de fonctions de forme de la figure 19 suggère que pour une vitesse réduite $\mathrm{V}^{*}$ donnée non nulle, $\left.\mathrm{a}_{\mathrm{j}}^{0} \mathrm{x}\right)$ se déduit de $\mathrm{a}^{\mathrm{o}}(\mathrm{x})$ (cas plastique) à l'aide d'une transformation géométrique à définir.

Une expression analytique inspirée de celle associée à $\mathrm{a}^{0}(\mathrm{x})$ et donnant satisfaction pour une large gamme de vitesses de creusement est proposée:

$$
a_{v p}^{0}(x)=1-\left(\frac{0,84}{\frac{x}{A R_{p}}+0,84}\right)^{28}
$$

$\mathrm{A}\left(\mathrm{V}^{*}\right)$

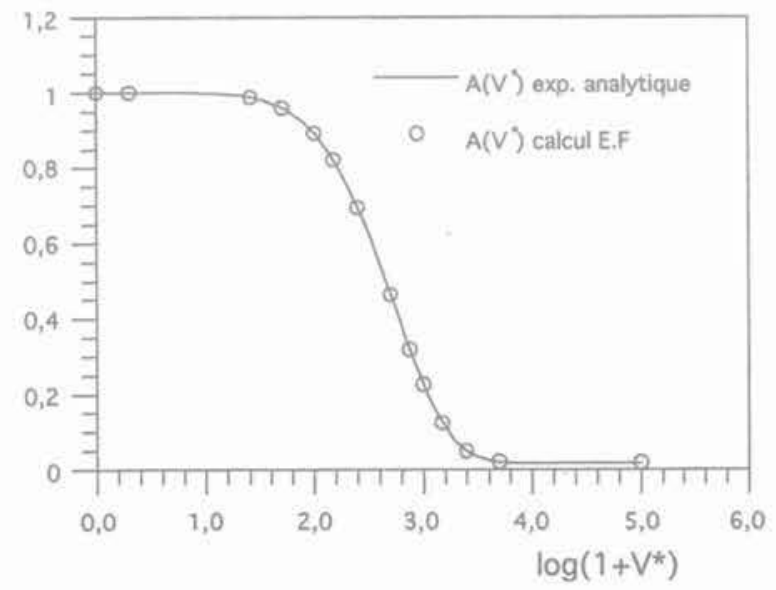

FiG. 20 Fonction $\mathrm{A}\left(\mathrm{V}^{*}\right)$. Function $\mathrm{A}\left(\mathrm{V}^{*}\right)$.

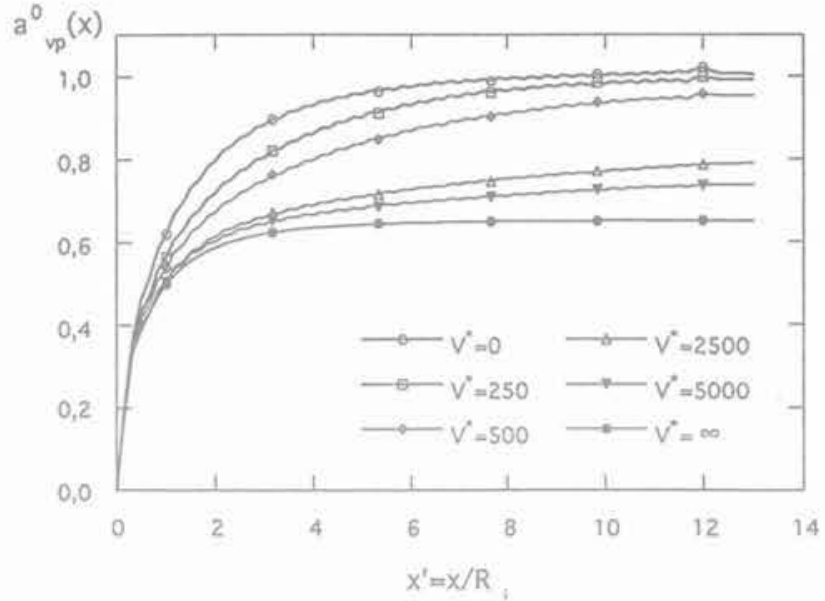

FIG. 19 Fonction de forme $\mathrm{a}_{\gamma_{\mathrm{p}}}^{0}(\mathrm{x})$ pour différentes vitesses.

Shape function $\mathrm{a}_{\mathrm{vp}}^{0}(\mathrm{x})$ for many tunnel advance rates:

oú $\mathrm{A}$ et $\mathrm{B}$ sont des paramètres tels que: $0<\mathrm{A} \leq 1$ et $0<B \leq 1$; on retrouve le cas non visqueux en posant à la limite $A=B=1, R_{n}$ est le rayon plastique du tunnel non soutenu à l'équilibre.

Pour déterminer les valeurs de $\mathrm{A}$ et $\mathrm{B}$, nous avons réalisé une étude paramétrique du tunnel non soutenu creusé dans un milieu viscoplastique en conditions axisymétriques, à l'aide de la méthode numérique d'activation/désactivation. L'étude porte sur une large gamme de valeurs de vitesses de creusement $\left(0 \leq V^{*} \leq\right.$ 5000 ) et de facteurs de chargement $1<\mathrm{Ns} \leq 5$. Elle a servi à confirmer la conjecture concernant l'expression analytique de $\mathrm{a}_{\mathrm{vp}}^{0}(\mathrm{x})$.

Les figures 20 et 21 illustrent les allures de variations de $A\left(V^{*}\right)$ et de $B\left(N, V^{*}\right)$ en fonction de $V^{*}$. Comme l'on peut observer sur ces figures, nous avons pu déterminer des expressions analytiques pour $\mathrm{A}$ et $\mathrm{B}$ qui calent parfaitement avec les valeurs tirées du calcul numérique. Ces expressions analytiques sont données en annexe 2.

A ne dépend pas de $N_{\text {c }}$ et est une fonction décroissante de $\mathrm{V}^{*}$ entre 0 et 1 : l'effet d'une augmentation de la vitesse de creusement se traduit donc par une affinité sur l'axe des abscisses, ce qui traduit le fait que plus V* est grand, plus le temps nécessaire à la stabilisation des convergences est grand.

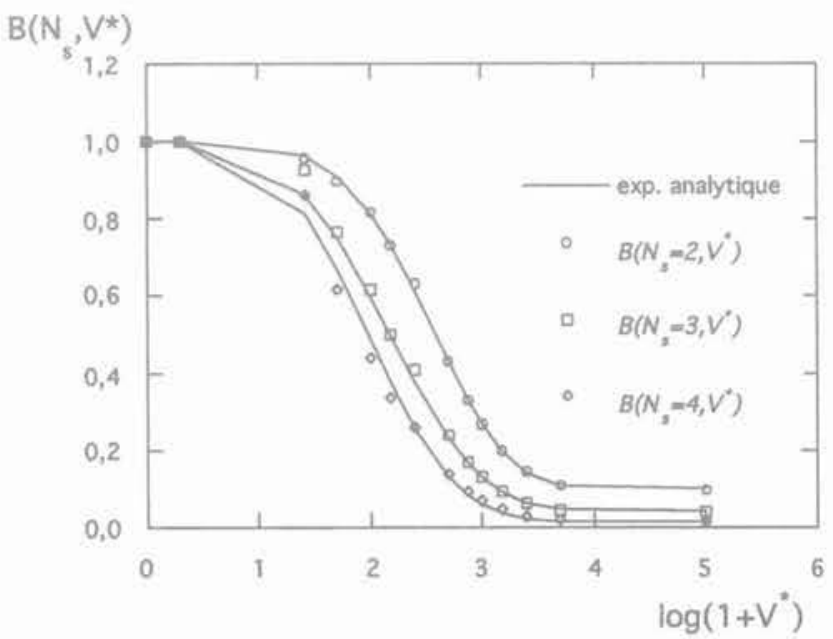

FIG. 21 Fonction $\mathrm{B}\left(\mathrm{N}_{\mathrm{G}}, \mathrm{V}^{*}\right)$ Function $\mathrm{B}\left(\mathrm{N}_{5}, \mathrm{~V}^{*}\right)$. 
$B$ est également une fonction décroissante de $V^{*}$ entre 0 et 1 . Mais ici, $B$ dépend également du paramètre de plasticité $N_{s}$. Cependant, en plasticité $\left(V^{*} \rightarrow 0\right)$ cette dépendance n'existe plus et on $\mathrm{a} B=1$.

En résumé, dans tous les cas, la fonction de forme du tunnel non soutenu a une expression simple et unique donnée par l'équation 24.

Remarques:

Pour tout $\mathrm{x}$, on $\mathrm{a}: 1-\mathrm{a}_{\mathrm{vp}}^{0}(\mathrm{x})=\left(1-\mathrm{a}^{0}\left(\frac{\mathrm{x}}{\mathrm{A}}\right)\right)^{\mathrm{B}}$ ce qui finit d'expliciter la nature de la transformation géométrique permettant de passer de $\mathrm{a}^{0}(\mathrm{x})$ à $\mathrm{a}_{\mathrm{vp}}^{0}(\mathrm{x})$.

Afin de construire la courbe de convergence $\mathrm{U}_{\mathrm{i}}(\mathrm{x})$ du tunnel non soutenu, on doit aussi déterminer la valeur de la convergence au front $\mathrm{Uf}^{f}(0)$.

Comme on pouvait s'y attendre, l'étude paramétrique nous a révélé que, en plus de $N_{s}, U^{\dagger}(0)$ dépend aussi de la vitesse réduite $V^{*}$ (Fig, 22), ce qui traduit un phénomène tout à fait naturel: pour un matériau donné, la convergence au front est d'autant plus faible que la vitesse est élevée. Une expression analytique "approchée » de $\mathrm{U}_{1}^{\mathrm{f}}(0)$ est proposée en annexe 2. Notons que cette expression vérifie les «conditions aux limites ) suivantes, pour tout N. (Corbetta, 1990; Bernaud, 1991):

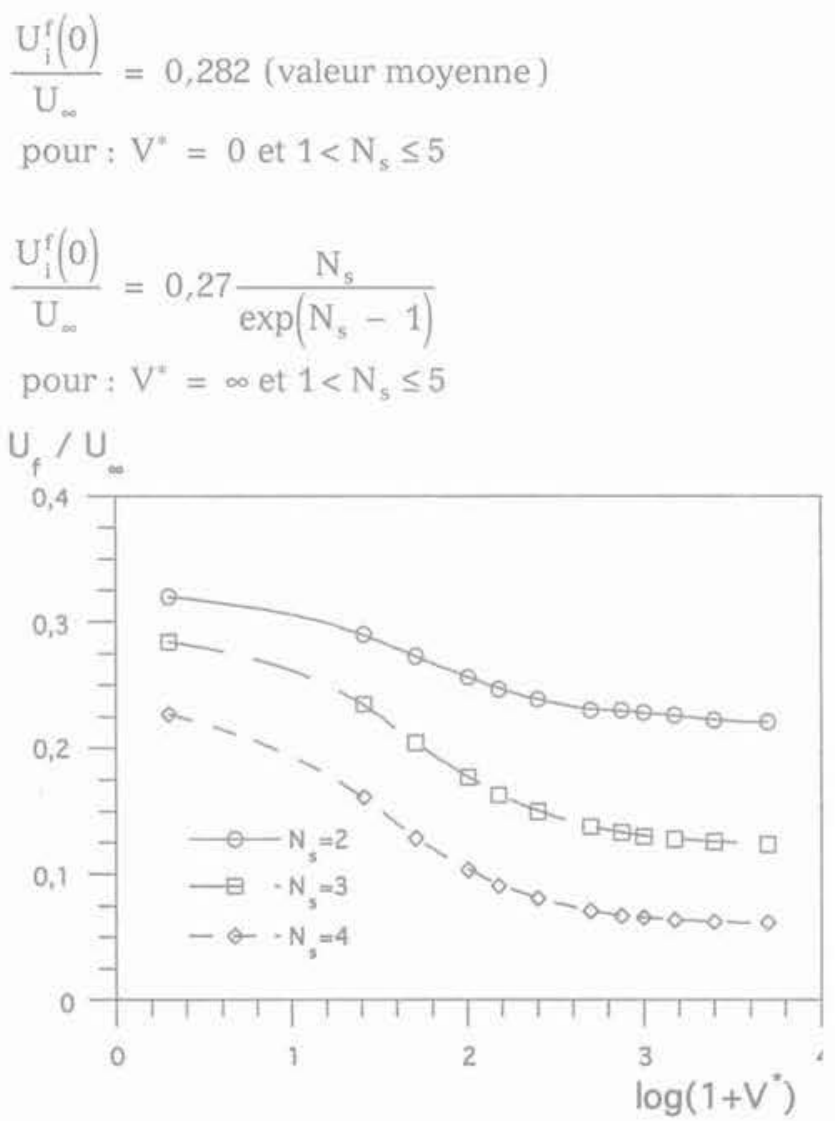

FG. 22 Convergence au front pour le tunnel non soutenu.

Convergence at the tunnel face for the unsupported tunnel.

- Conclusion de l'étude du tunnel non soutenu

Dans le cas du problème du tunnel non soutenu creusé dans un massif viscoplastique avec une vitesse constante, nous avons déterminé une expression ana- lytique pour la fonction de forme $\mathrm{a}_{\mathrm{vp}}^{0}(\mathrm{x})$ tout à fait satisfaisante. La convergence en paroi du tunnel en toute section x s'exprime par:

$$
\mathrm{U}_{i}^{f}(\mathrm{x})=\mathrm{a}_{\mathrm{vp}}^{0}(\mathrm{x})\left(\mathrm{U}_{\infty}-\mathrm{U}_{1}^{f}(0)\right)+\mathrm{U}_{1}^{f}(0
$$

La figure 23 montre la courbe de convergence (cas avec $\mathrm{N}_{\mathrm{s}}=2.5 ; \mathrm{V}^{*}=2500 ; \mathrm{E}^{*}=250$ ) tirée d'un calcul numérique axisymétrique et son estimation déduite de la formule ci-dessus. On constate la bonne concordance entre le calcul numérique et la méthode approchée.

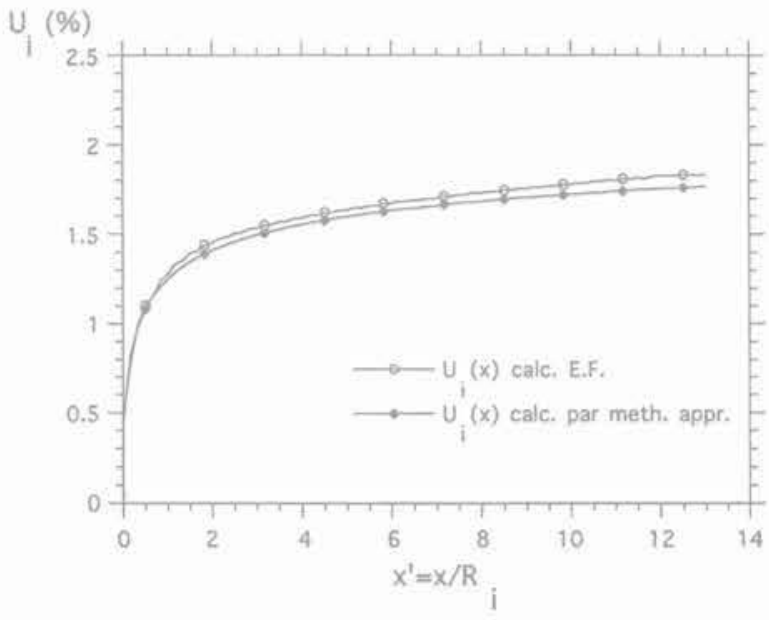

FG. 23 Approximation de $\mathrm{U}_{1}^{f}$ à I'aide de l'équation (25) Calculation of $\mathrm{U}_{i}^{+}(\mathrm{x})$ with the equation (25).

\section{T.1. $5,3,2$}

\section{Tunnel soutenu}

Toujours dans le même esprit lié aux principes de base de la nouvelle méthode implicite, la fonction de forme du tunnel soutenu est définie de la même manière qu'en élasticité et en plasticité par:

$$
a_{v p}^{s}(x)=\frac{U_{1}(x)-U_{p}}{U_{e q}-U_{f}}
$$

La fonction de forme $a_{v p}^{s}(x)$ doit tenir compte de la raideur du soutènement.

De même qu'en élasticité et en plasticité, bien que $a_{v p}^{s}(x)$ soit toujours une fonction croissante de $x$ entre 0 et 1 , les calculs numériques du tunnel soutenu montrent que la variation de $a_{\mathrm{vp}}^{\mathrm{s}}(\mathrm{x})$ est d'autant plus prononcée que la raideur du soutènement est élevée (toutes grandeurs égales par ailleurs). Ce phénomène est illustré sur la figure 24 où nous avons tracé les valeurs de $a_{\text {va }}^{5}(\mathrm{x})$ obtenues des calculs numériques pour différents $\mathrm{K}_{\mathrm{s}}^{\prime}$ dans le cas où $\mathrm{N}_{\mathrm{s}}=2 ; \mathrm{V}^{*}=500$; $E^{*}=250 ; d_{0}^{\prime}=1$.

De façon naturelle dans le cadre de la Nouvelle Méthode Implicite, on est donc amené à poser:

$$
a_{v p}^{s}(x)=a_{v p}^{0}(\alpha x)
$$

Ce qui semble traduire le phénomène observé sur la figure 24: pour $\mathrm{N}_{\text {s }}$ et $\mathrm{V}^{*}$ donnés, les courbes $\mathrm{a}_{\mathrm{v}}^{\mathrm{s}}(\mathrm{x})$ se déduisent les unes des autres par simple affinité de rapport $\alpha$. 


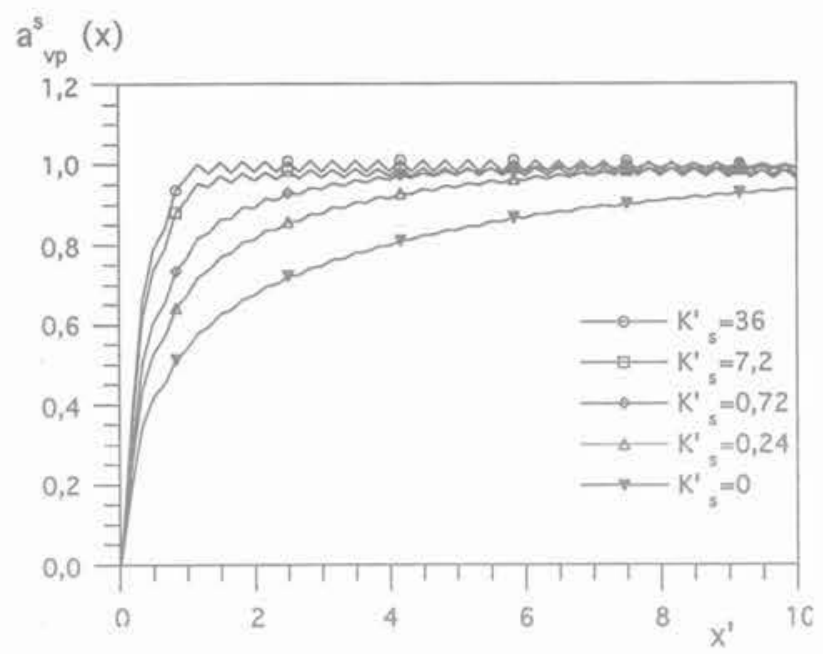

FIG. 24 Fonction $\mathrm{a}^{\mathrm{s}}(\mathrm{x})$ pour différents $\mathrm{K}_{\text {, }}$, Eunction $\mathrm{a}_{v p}^{\mathrm{s}}(\mathrm{x})$ form many $\mathrm{K}_{\text {: }}$.

Par contre, si en élasticité et en plasticité, le paramètre $\alpha^{*}=\alpha R / R$ ne dépend principalement que de $K^{\prime}$ en viscoplasticite, on pose a priori: $\alpha^{*}=\alpha^{*}\left(\mathrm{~N}_{s^{\prime}}, V^{*}, \mathrm{~K}_{5}^{\prime}\right)$.

Pour déterminer cette fonction $\alpha^{*}$ nous avons réalisé plusieurs calculs numériques axisymétriques du tunnel soutenu couvrant une large gamme de paramètres:

$\mathrm{N}_{\mathrm{s}}=1$ à $5, \mathrm{~V}^{*}=0$ à $5000, \mathrm{~K}_{\mathrm{s}}^{\prime}=0,07$ à $72, \mathrm{~d}_{0}^{\prime}=1 / 3$ à 1 .

D'autre part, nous avons concentré les efforts de calage à la fois pour les $x$ faibles $(0 \leq x \leq 2 R)$ et pour les $x$ grands (stabilité de la convergence loin du front de taille) sans se soucier des $\mathrm{x}$ intermédiaires.

Les premiers résultats ont montré que $\alpha^{*}$ peut prendre de grandes valeurs selon que la rigidité du soutènement, que le facteur de charge et que la vitesse réduite de creusement sont grands, ce qui nous a conduit à utiliser des échelles logarithmiques. En plasticité (pour notre critère) on a ;

$$
\alpha^{*}=\alpha \frac{R_{i}}{R_{p}}=1,82 \sqrt{K_{s}^{\prime}}
$$

$c^{\prime}$ est-à-dire $\log \left(\alpha^{*}\right)=0,5 \log \left(K_{s}^{\prime}\right)+0,6$.

Autrement dit, en plasticité, il existe une relation linéaire entre $\log \left(\alpha^{*}\right)$ et $\log \left(\mathrm{K}^{\prime}\right)$. Notre étude paramétrique nous a montré que cette linéarité se maintient pour tous les couples $\left(\mathrm{V}^{*}, \mathrm{~N}_{\mathrm{s}}\right.$ ) étudiés. On est donc amené à poser, a priori:

$$
\log \left(\alpha^{*}\right)=C_{1}\left(N_{s^{\prime}}, V^{*}\right) . \log \left(K_{s}^{\prime}\right)+C_{2}\left(N_{s^{\prime}} V^{*}\right)
$$

avec les conditions ( initiales» sur $\mathrm{C}_{1}$ et $\mathrm{C}_{2}$ : $\mathrm{C}_{1}\left(\mathrm{~N}_{\mathrm{s}^{\prime}} \mathrm{V}^{*}=0\right)=0,5$ et $\mathrm{C}_{2}\left(\mathrm{~N}_{\mathrm{s}^{\prime}} \mathrm{V}^{*}=0\right)=0,6$.

Nous avons déterminé des expressions analytiques satisfaisantes pour $\mathrm{C}_{1}\left(\mathrm{~N}_{s^{\prime}} \mathrm{V}^{*}\right)$ et $\mathrm{C}_{2}\left(\mathrm{~N}_{\mathrm{s}^{\prime}} \mathrm{V}^{*}\right)$ (données dans l'annexe 2).

En résumé, il est intéressant de constater qu'en viscoplasticité on peut aussi utiliser le même type d'affinité entre la fonction de forme du tunnel non soutenu et celle du tunnel soutenu. D'autre part, même si le rapport d'affinité $\alpha$ du tunnel viscoplastique s'exprime d'une façon plus complexe que pour les lois de comportement qui ne dépendent pas du temps, son expression reste encore simple compte tenu de la complexité du problème étudié:

$$
\alpha^{*}=\alpha \frac{R_{1}}{R_{p}}=K_{s}^{\prime C_{1}} \exp \left(C_{2}\right)
$$

où : $\mathrm{C}_{1}=\mathrm{C}_{1}\left(\mathrm{~N}_{\mathrm{s}^{\prime}}, \mathrm{V}^{*}\right)$ et $\mathrm{C}_{2}=\mathrm{C}_{2}\left(\mathrm{~N}_{\mathrm{s}^{\prime}}, \mathrm{V}^{*}\right)$

Un autre paramètre nécessaire au développement de cette nouvelle méthode est la convergence au front du tunnel soutenu.

De même qu'en élasticité et en plasticité, dans le cas viscoplastique, à $\mathrm{N}_{\text {s }}$ et $\mathrm{V}^{*}$ fixés, la variation de $\mathrm{U}_{\text {f }}$ en fonction de $\mathrm{K}_{\mathrm{s}}^{\prime}$ est très faible. La convergence du tunnel soutenu sera donc considérée comme indépendante de $\mathrm{K}^{\prime}$, cependant son expression analytique ( approchée» en tiendra implicitement compte et sera donc différente de celle du tunnel non soutenu $\mathrm{U}^{\dagger}(0)$ (voir annexe 2). Notons que $U$, vérifie les «conditions aux limites ) suivantes (cas d'élasticité et plasticité données précédemment):

$$
\begin{aligned}
& \frac{\mathrm{U}_{t}\left(\mathrm{~N}_{\mathrm{s}}, \mathrm{V}^{*}=0\right)}{\mathrm{U}_{-}}=0,413-0,0627 \mathrm{~N}_{\mathrm{s}} \\
& \text { et } \frac{\mathrm{U}_{\mathrm{f}}\left(\mathrm{N}_{\mathrm{s}}, \mathrm{V}^{*}=\infty\right)}{\mathrm{U}_{\infty}}=0,27 \frac{\mathrm{N}_{\mathrm{s}}}{\exp \left(\mathrm{N}_{\mathrm{s}}-1\right)}
\end{aligned}
$$

En résumé, l'application de notre méthode au cas viscoplastique consiste à se donner:

- l'expression analytique de la fonction de forme du tunnel non soutenu $a_{\mathrm{vp}}^{0}(\mathrm{x})$;

- l'expression (eq.30) de la fonction de soutènement $\alpha^{*}=\alpha^{*}\left(\mathrm{~N}_{\mathrm{s}^{\prime}} \mathrm{V}^{*}, \mathrm{~K}_{\mathrm{g}}^{\prime}\right)$;

- l'expression de la convergence au front de taille $U_{f}$

A partir de ces données, le principe d'application général de la nouvelle méthode implicite est toujours le même : la solution cherchée $\left(\mathrm{P}_{\mathrm{eq}}, \mathrm{U}_{\mathrm{eq}}\right)$ est à l'intersection des deux courbes CV (courbe de convergence à long terme) et CF (courbe de confinement) :

$$
\left\{\begin{array}{l}
\mathrm{P}_{\mathrm{eq}}=\mathrm{CV}\left(\mathrm{U}_{\mathrm{eq}}\right) \\
\mathrm{P}_{\mathrm{eq}}=\mathrm{CF}\left(\mathrm{U}_{\mathrm{eq}}-\mathrm{U}_{0}\right)
\end{array}\right.
$$

où $\mathrm{U}_{0}$ désigne la convergence en paroi lors de la pose du soutènement:

$$
\mathrm{U}_{0}=\mathrm{a}_{\mathrm{vp}}^{\mathrm{s}}\left(\mathrm{d}_{0}\right)\left(\mathrm{U}_{\mathrm{eq}}-\mathrm{U}_{\mathrm{f}}\right)+\mathrm{U}_{\mathrm{f}}
$$

On observe que dans les milieux viscoplastiques étudiés ici, la convergence $\mathrm{U}_{0}$ qui est le paramètre clé du problème d'interaction massif-soutènement, $s^{\prime}$ exprime par une fonction du type $\left.\mathrm{U}_{0}=\mathrm{f}_{\left(\mathrm{d}_{0}^{\prime}\right.} \mathrm{N}_{\mathrm{s}^{\prime}} \mathrm{V}^{*}, \mathrm{~K}_{s}^{\prime}\right)$.

En viscoplasticité, la nouvelle méthode implicite a été aussi développée pour un matériau avec un critère de Coulomb et un potentiel de Tresca. Dans ce cas la fonction de soutènement $\alpha^{*}=\alpha^{*}\left(\mathrm{~N}_{\mathrm{s}^{\prime}} \mathrm{V}^{*}, \mathrm{~K}_{\mathrm{s}}^{\prime}\right)$ obtenue $\mathrm{s}^{\prime}$ exprime par la somme de deux termes : le premier est celui correspondant au critère de Tresca (eq.30) et le deuxième est donné par une fonction simple qui dépend de l'angle de frottement.

\section{4}

\section{Résultats et validation}

Compte tenu du nombre important de paramètres intervenant dans l'étude et de leur grande dépendance, les formules analytiques approchées sont très satisfai- 
santes au cas par cas, mais conduisent à des valeurs de $\alpha$ approximatives dans beaucoup de cas.

Cependant, en appliquant la méthode ainsi présentée, qui de plus est facile à programmer à partir des formules fournies en annexe, on a relevé que l'erreur moyenne commise sur $\mathrm{U}_{\text {eq }}$ et $\mathrm{P}_{\text {eq }}$ (par rapport au calcul E.F. axisymétrique) ne dépasse pas $15 \%$ en valeur absolue.

Dans le tableau 2, on illustre quelques résultats du problème des tunnels creusés dans les milieux viscoplastiques (matériau de Tresca).

Les figures 25 a et $25 \mathrm{~b}$ montrent l'influence de la vitesse de creusement $V^{*}$ sur la convergence à l'instant de pose $\mathrm{U}_{0}$ ainsi que sur la pression à l'équilibre $\mathrm{P}_{\mathrm{eq}}$ pour le problème avec $\mathrm{N}_{\mathrm{s}}=2 ; \mathrm{K}_{\mathrm{s}}^{\prime}=2,4$.

Ces exemples montrent encore une fois l'importance de la vitesse de creusement sur l'équilibre de l'ouvrage: pour une distance de pose d donnée la pression à l'équilibre peut être multipliée par deux. lorsqu'on passe d'une vitesse faible à une autre très élevée.

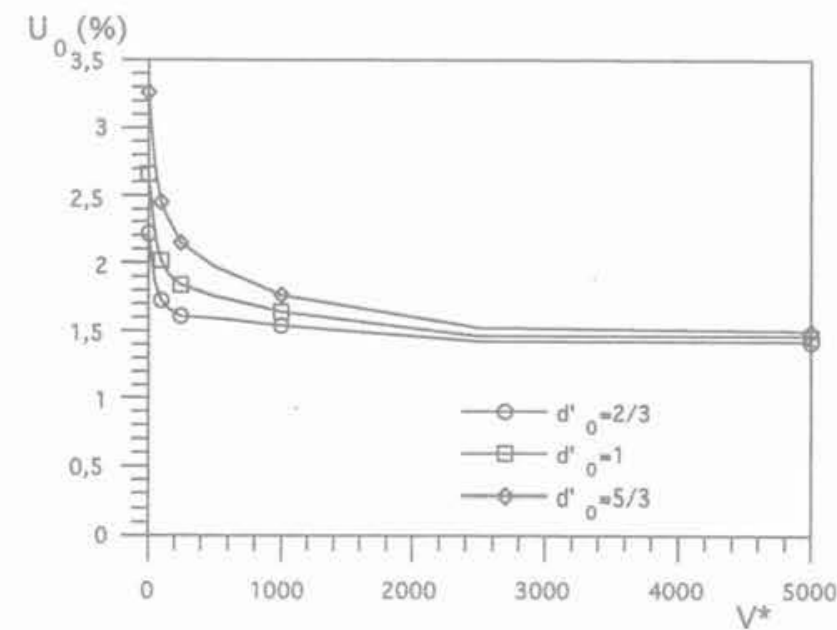

FIG. 25a Variation de $U_{0}$ en fonction de $V^{*}$. $\mathrm{U}_{6}$ versus $\mathrm{V}^{*}$ for many $\mathrm{d}_{0}$

\section{6}

\section{Application à l'étude d'un problème en déformation plane}

La méthode convergence-confinement permet, en pratique, d'avoir recours à une approche simplifiée en déformation plane grâce à la notion de "pression fictiven (notée P') s'exerçant sur la paroi du tunnel et rendant compte de la proximité du front de taille (voir figure 15).

Souvent, dans le cas de la viscoplasticité, les calculs 2D en déformation plane sont réalisés loin du front de taille (pression fictive nulle) en tenant compte uniquement de la pression de soutènement (Sharma et al.; 1985). Si en élastoplasticité Pf a été reliée à l'avancement du front de taille de manière satisfaisante (Bernaud et Rousset, 1992; Nguyen-Minh et Guo, 1993a), en viscoplasticité le problème reste posé malgré quelques approches (Sakurai, 1978).

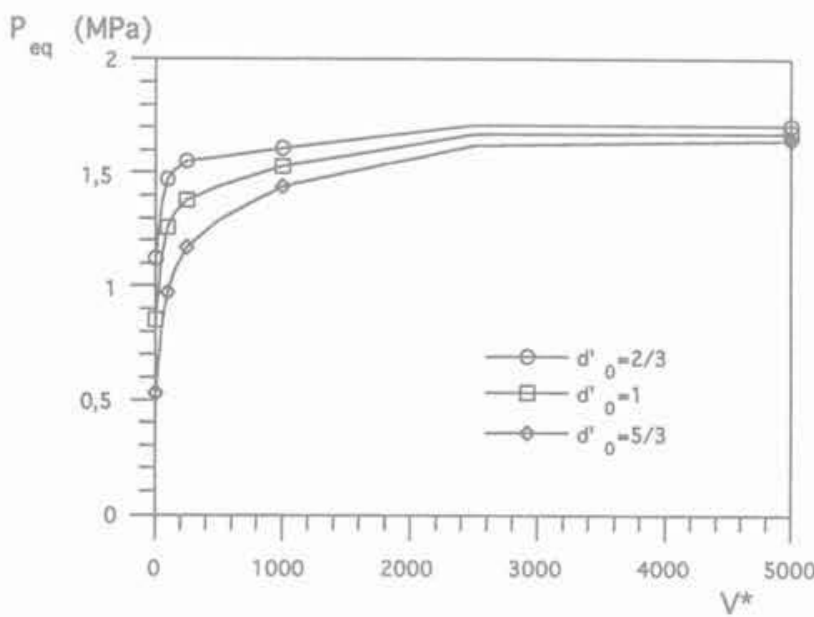

FIG. 25b Variation de $P_{\text {eq }}$ en fonction de $V^{\star}$. $P_{e q}$ versus $V^{*}$ for many $d_{0}$

TABLEAU II Validation de la Nouvelle Méthode Implicite en viscoplasticité Validity of the New Implicit Method for viscoplasticity.

\begin{tabular}{|c|c|c|c|c|c|c|c|}
\hline$N_{s}$ & $d{ }_{0}$ & $V^{*}$ & $\mathrm{~K}_{\text {s }}$ & $\mathrm{H}_{\mathrm{eq}}$ E.F. $\%$ & $\Delta \mathrm{U}_{\mathrm{xd}} / \mathrm{U}_{\mathrm{eq}} \%$ & $P_{\text {oq }}$ E.F. (MPa) & $\Delta \mathrm{P}_{\text {eq }} / \mathrm{P}_{\text {eqf }}$ \\
\hline \multirow{5}{*}{3} & \multirow{5}{*}{1} & 150 & 0,24 & 2,80 & 0 & 0,92 & 0 \\
\hline & & 150 & 24 & 2,00 & 7 & 1,59 & -9 \\
\hline & & 500 & 0,72 & 2,10 & -2 & 1,49 & 3 \\
\hline & & 500 & 7,2 & 1,66 & 10 & 1,97 & -11 \\
\hline & & 5000 & 24 & 1,48 & 11 & 2,19 & -11 \\
\hline 4 & 1 & 500 & 24 & 2,20 & 19 & 3,40 & -12 \\
\hline \multirow[t]{2}{*}{2} & 1 & 1000 & 36 & 0,38 & 4 & 0,72 & -9 \\
\hline & $1 / 3$ & 1000 & 0,72 & 1,06 & -5 & 0,86 & 11 \\
\hline 3 & $1 / 3$ & 2000 & 18 & 0,39 & -1 & 1,78 & 1 \\
\hline \multirow[t]{2}{*}{5} & 1 & 500 & 0,72 & 1,93 & 8 & 1,53 & -4 \\
\hline & 1 & 150 & 0,72 & 2,41 & 19 & 1,36 & -12 \\
\hline
\end{tabular}


Pour le calcul 1D d'un tunnel creusé en milieu viscoplastique de Bingham, la solution semi-analytique est classique (Rousset, 1988; Nguyen-Minh, 1986; Yamatomi et al., 1993). Dans le cas particulier d'un matériau incompressible $(\nu=0,5)$ de Tresca, on a :

$$
\frac{E^{\prime}}{2 C} \dot{U}_{i}=-\frac{E^{\prime}}{\eta} \log \left(\frac{E^{\prime}}{2 C} U_{i}\right)-\frac{\dot{P}_{i}}{C}+\frac{E^{\prime}}{\eta} \frac{P_{\infty}-P_{i}-C}{C}
$$

où $\mathrm{P}_{\text {f }}$ : pression appliquée à la paroí.

$\underset{\dot{X}}{U_{j}}$ : convergence à la paroi.

$\dot{X}$ : désigne $\mathrm{dX} / \mathrm{dt}$.

$$
E^{\prime}=\frac{E}{1-v^{2}}=\frac{4}{3} E
$$

La formule (25) qui donne une expression analytique approchée de la convergence du tunnel non soutenu nous permet, en l'introduisant dans l'équation (33) (en remplaçant x par Vt car V est constante) d'obtenir une équation différentielle du premier ordre sur la fonction inconnue $\mathrm{P}(\mathrm{t})$.

Pour un tunnel non soutenu, $\mathrm{P}(\mathrm{t})$ représente la pression fictive $P^{\prime}(t)$ s'exerçant sur la paroi du tunnel et rendant compte de la proximité du front de taille $(\mathrm{x}=\mathrm{Vt})$. Ce concept s'insère donc dans le cadre de la méthode convergence-confinement et permet le passage 3D - 2D déformation plane dans les hypothèses d'axisymétrie.

Le nouveau système:

$$
\left\{\begin{array}{l}
U_{i}(t)=a_{v p}^{0}(V t)\left(U_{\infty}-U_{i}^{\prime}(0)\right)+U_{i}^{f}(0) \\
\frac{\dot{P}_{i}^{f}}{C}-\frac{E^{\prime}}{\eta} \frac{P_{\infty}-P_{i}^{f}-C}{C}=-\frac{E^{\prime}}{2 C} \dot{U}_{j}(t)-\frac{E^{\prime}}{\eta} \log \left(\frac{E^{\prime}}{2 C} U_{j}(t)\right)
\end{array}\right.
$$

pour $\mathrm{P}_{1}^{f}<\mathrm{P}_{\mathrm{ie}}$ avec $\mathrm{P}_{\mathrm{ie}}=\mathrm{P}_{-}-\mathrm{C}$

nous permet après résolution, de tracer la courbe (t, P) correspondant à une vitesse $\mathrm{V}$ de creusement donnée (figure 26),

A partir de là, une analogie avec la notion de taux de déconfinement lors de pose du soutènement $\left(\lambda_{0}\right)$ introduite auparavant en élastoplasticité s'impose. La connais-

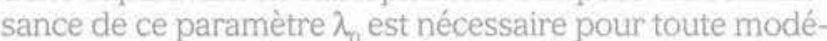
lisation du tunnel soutenu 2D en déformation plane.

Dans notre cas, en viscoplasticité, un calcul de prédimensionnement supposant les hypothèses d'axisymétrie satisfaites et utilisant les calculs de la nouvelle méthode implicite nous fournit une valeur de la convergence à la paroi lors de la pose du soutènement $\mathrm{U}_{0}=$ $\mathrm{U}_{0}\left(\mathrm{~N}_{\mathrm{s}^{\prime}} \mathrm{d}_{\mathrm{y}^{\prime}}^{\prime} \mathrm{K}_{\mathrm{s}^{\prime}}^{\prime}, V^{*}\right)$. A cette dernière valeur correspond un temps de pose $t_{0}$ fictif (puisque différent de $\mathrm{d}_{0} \mathrm{~V}$ ) qu'on reporte sur l'axe des abscisses de la figure 26 et qui nous fournit la valeur de $\lambda_{0}=\lambda\left(t_{0}\right)$ correspondante.

L'intérêt d'un calcul 2D en déformation plane est de pouvoir étudier des géométries et des chargements complexes (section en forme de fer à cheval, chargement anisotrope...).

Désormais, pour réaliser un calcul 2D en déformation plane dans un massif viscoplastique, on pourra

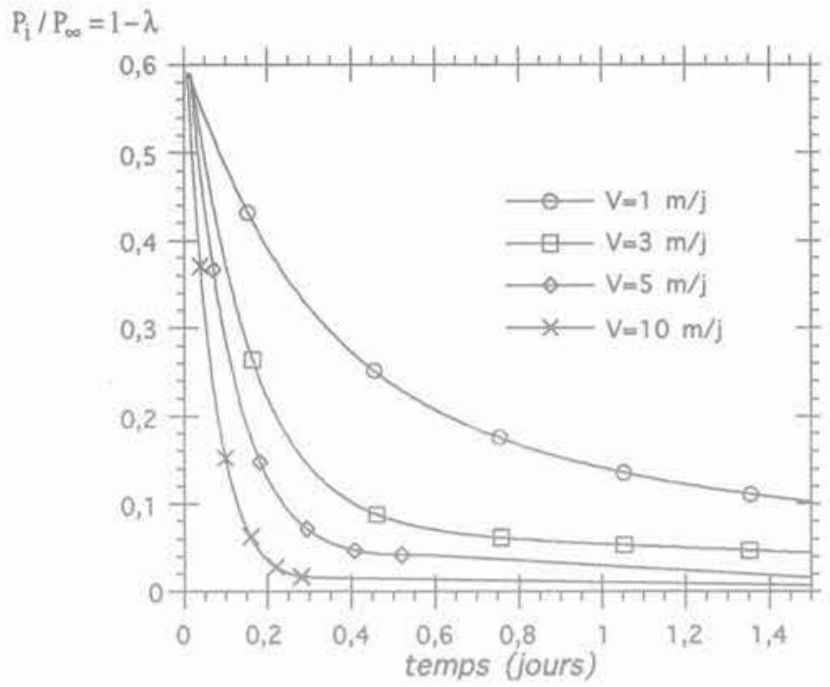

FIG. 26 Pression fictive en fonction du temps pour plusieurs vitesses de creusement. $(\mathrm{P} \infty=5 \mathrm{MPa}, \mathrm{C}=2 \mathrm{MPa})$

Fictitious pressure versus time for many tunnel adance rates.

rendre compte avec une bonne précision de la proximité du front de taille lors de la pose du soutènement en tenant compte de la pression fictive.

\section{7}

\section{Conclusions}

Dans cet article on a exposé les derniers développements de la «Nouvelle Méthode Implicite » pour le dimensionnement des tunnels profonds revêtus creusés dans des milieux élastoplastiques et élastoviscoplastiques.

Cette méthode a les mêmes principes de base de la méthode convergence-confinement, mais permet de bien tenir compte du caractère tridimensionnel et couplé du problème du tunnel profond soutenu. En particulier la « Nouvelle Méthode Implicite » tient compte de la rigidité du soutènement dans le calcul de la convergence à l'instant de pose du soutènement $\mathrm{U}_{0}$ (ou, taux de déconfinement à la pose $\lambda$ ), qui est le paramètre fondamental du problème d'interaction.

La précision de la méthode a été testée en comparant avec des résultats de calculs numériques axisymétriques et l'erreur moyenne commise sur les paramètres dimensionnants du tunnel $P_{\text {eq }}$ et $U_{\text {eq }}$ est inférieure à $10 \%$ en valeur absolue (pour l'ensemble des lois de comportements étudiées), ce qui nous semble très satisfaisant pour une étude géotechnique.

De plus, la « Nouvelle Méthode Implicite », tout en tenant compte de la complexité du problème de couplage massif-soutènement, est souple d'emploi et peut être facilement intégrée dans un logiciel de dimensionement des tunnels.

\section{Bibliographie}

AFTES (1983) - Recommandations sur l'emploi de la méthode ConvergenceConfinement. Tunnels et Ouvrages souterrains, $n^{\circ} 59$, septembre-octobre.
Bernaud D, (1991) - Tunnels profonds dans les milieux viscoplastiques. Approche expérimentale et numérique. Thèse, École Nationale des Ponts et Chaussées.
Bernaud D, Rousset G. (1992) - La nouvelle méthode implicite pour l'étude du dimensionnement des tunnels, Revue Française de Géotechnique, n60, $3^{\circ}$ trim., pp. 5-26. 
Bernaud D., Rousset G. (1994) - New Implicit Method for tunnel analysis: application to deep tunnels in clay. ISRM Int Symp., Integral Approach to Applied Rock Mechanics, Santiago, vol. 1, pp. 361-371

Corbetta F. (1990) - Nouvelles méthodes d'étude des tunnels profonds. Calculs analytique et numérique, thèse, Ecole Nationale Supérieure des Mines de Paris.

Hanafy E.A., Emery J.J. (1982) - Advancing face simulation of tunnel excavations and lining placement. Proc, 13th Canadian Symp. Rock Mechanics : Underground Rock Engineering, Toronto. pp. 119-125.

Hoek E., Brown E.T. (1988) - The HoekBrown failure criterion - a 1988 update' Proc. of the 15th Canadian Rock Mechanics Symposium, pp, 31-38.

Hoek E Wood D Shah S (1992) - A modified Hoek-Brown failure criterion for jointed rock masses, Eurock'92, pp. 209-214.

Nguyen-Minh D. (1986) - Modèle rhéologique pour l'analyse du comportement différé des galeries profondes, Proc. Int. Cong. «Large Underground Openings w, Florence, vol. 2, pp. 659
Nguyen-Minh D., Guo C. (1993a) - Sur un principe d'interaction massif-soutènement des tunnels en avancement stationnaire, Eurock'93, Lisbonne, Portugal.

Nguyen-Minh D., Guo C. (1993b) - Tunnels creusés en milieu viscoplastique. Géotechnique et Environnement, Colloque Franco-Polonais, Nancy, 15-19 nov.

Pan X.D., Hudson J.A.(1989) - Plane strain analysis in modelling three-dimensional finite element method, Int. J. Rock Mechanics \& Mining Sciences, 25.

Panet M. Guellec P. (1974) - Contribution à l'étude du soutènement d'un tunnel à l'arrière du front de taille. Proc. 3rd Int. Cong. Rock Mechanics, Denver, vol. IIB.

Panet M., Guenot A. (1982) - Analysis of convergence behind the face of a tun nel, Proc. Int. Symp. a Tunnelling 82 , Brighton.

Perzyna (1966) - Fundamental problems in viscoplasticity, Advances in Applied Mechanics, vol. 9.

Ranken R.E., Ghaboussi J. (1975) - Tunnel design considerations: analysis of stresses and deformations around advancing tunnels, Report UILU-ENG 75-2016, National Technical Information Service, Springfield, USA.
Rousset G. (1988) - Comportement mécanique des argiles profondes: application au stockage de déchets radioactifs, Thèse, École Nationale des Ponts et Chaussées.

Sakurai S. (1978) - Approximate timedependent analysis of tunnel supports structure considering progress of tunnel face, Int. J. Numerical \& Analytical Methods in Geomechanics, vol. II, $n^{\circ} 2$.

Sharma K.G., Varadarajan A., Srivastava R.K. (1985) - Elasto-viscoplastic finite element analyses of tunnels, 5 th Int. Conf. on Num. Meth. in Geomech. Nagoya, pp. 1141-1148.

Yamatomi J, Yamashita S., Beniawski ZT, Ogata Y, Kawabe K. (1993) - An analytical method of stress and displacement around a circular tunnel excavated in rock mass with non-linear time-dependency, Eurock'93. Lisbonne, Portugal

Zienkiewickz O.C., Cormeau 1.C. $1974-$ Visco-Plasticity and Creep in Elastic Solids-A Unified Numerical Solution Approach, Int. J, Num. Meth. Engng, vol. VIII, pp. 821-845.

\section{ANNEXE 1}

\section{Convergence du tunnel non soutenu}

Matériau élastiquement incompressible $(\nu=0,5)$

- Tresca : $\mathrm{U}_{\infty}=\frac{1,5}{\mathrm{E}} \mathrm{C} \exp \left(\mathrm{N}_{\mathrm{s}}-1\right)$

- Coulomb :

$U_{-}=\frac{1,5}{E} \frac{K_{p}-1}{K_{p}+1}\left(P_{\sim}+H\right)\left\{\frac{2}{K_{p}+1} \frac{P_{\infty}+H}{H}\right\}^{2 /\left(K_{p}-1\right)}$

avec $\mathrm{H}=\mathrm{C} \operatorname{cotg} \phi$

- Hoek-Brown:

$\mathrm{U}_{\alpha}=\frac{0,75}{\mathrm{E}} \sqrt{\sigma_{c}^{2}-m \sigma_{c} \sigma_{y}} \exp \left\{\frac{-4\left(\sigma_{c}-\sqrt{\sigma_{c}^{2}-m \sigma_{c} \sigma_{y}}\right)}{m \sigma_{c}}\right\}$

- Hoek-Brown Modifié :

$U_{\infty}=\frac{0,75}{E} \sigma_{c}\left(-m_{b} \frac{\sigma_{y}}{\sigma_{c}}\right)^{a} \exp \left\{\frac{2}{(1-a) m_{b}}\left(-m_{b} \frac{\sigma_{y}}{\sigma_{c}}\right)^{1-\mathrm{a}}\right\}$

\section{Solution du problème plan à l'équilibre}

a) Élasticité

Dans ce cas la solution du système (8) est explicite:

$$
\begin{gathered}
U_{e q}=\frac{P_{\infty}+K_{s} U_{f}\left(1-a^{s}\left(d_{0}\right)\right)}{K_{s}\left(1-a^{s}\left(d_{0}\right)\right)+2 \frac{E}{3}} \\
P_{e q}=P_{\infty}-\frac{2}{3} E U_{e q}
\end{gathered}
$$

b) Plasticité Tresca (pour $\mathrm{P}_{\text {eq }}<\mathrm{P}_{-}-\mathrm{C}$; pour $\mathrm{P}_{\mathrm{eq}}>\mathrm{P}_{-}-\mathrm{C}$ le massif est élastique)

La solution du système (8) équivaut à la résolution de l'équation:

$$
\mathrm{a} \log \mathrm{U}_{\mathrm{eq}}+\mathrm{b} \mathrm{U}_{\mathrm{eq}}+\mathrm{c}=0
$$

avec:

$$
\left\{\begin{array}{l}
\mathrm{a}=-\frac{\mathrm{C}}{\mathrm{K}_{\mathrm{s}}} \\
\mathrm{b}=\mathrm{a}^{5}\left(\mathrm{~d}_{0}\right)-1 \\
\mathrm{c}=\left(1-\mathrm{a}^{5}\left(\mathrm{~d}_{0}\right)\right) \mathrm{U}_{\mathrm{f}}+\frac{\mathrm{P}_{-}}{\mathrm{K}_{\mathrm{s}}}+\frac{\mathrm{C}}{\mathrm{K}_{\mathrm{s}}}\left\{\log \left(\frac{1,5 \mathrm{C}}{\mathrm{E}}\right)-1\right\}
\end{array}\right.
$$

Pour Mises $\left(F=\sqrt{3 J_{2}}-2 C\right)$ la solution du système (8) est obtenue en remplaçant C par $2 \mathrm{C} / \sqrt{3}$.

\section{c) Plasticité Coulomb}

(pour $\mathrm{P}_{\mathrm{eq}}<\frac{2}{\mathrm{~K}_{\mathrm{p}}+1}\left\{\mathrm{P}_{\infty}+\frac{\mathrm{H}}{2}\left(1-\mathrm{K}_{\mathrm{p}}\right)\right\}$, sinon

le massif est élastique )

La solution du système (8) équivaut à la résolution de l'équation:

$$
a U_{e q}^{n_{1}}+b U_{\text {eq }}^{n_{2}}+c=0
$$


avec :

$$
\left\{\begin{array}{l}
a=\left\{\frac{\left(K_{p}+1\right) E}{1,5\left(K_{p}-1\right)\left(P_{\infty}+H\right)}\right\}^{\left(K_{p}-1\right) / 2}\left(\frac{K_{p}+1}{2}\right) \frac{K_{s}}{E}\left(1-a^{5}\left(d_{0}\right)\right) \\
b=\left\{\frac{\left(K_{p}+1\right) E}{1,5\left(K_{p}-1\right)\left(P_{\infty}+H\right)}\right\}^{\left(K_{p}-1\right) / 2}\left(\frac{K_{p}+1}{2}\right) \\
c=-\frac{\left(P_{\infty}+H\right)}{E} \\
\left.U_{f} \frac{K_{s}}{E}\left(a^{5}\left(d_{0}\right)-1\right)+\frac{H}{E}\right\} \\
n_{1}=\frac{K_{p}+1}{2} \\
n_{2}=\frac{K_{p}-1}{2}
\end{array}\right.
$$

d) Plasticité Hoek-Brown (pour $P_{\text {eq }}<\left|\sigma_{y}\right|$, sinon la roche est élastique)

La solution du problème est donnée par;

$$
a\left(\log U_{e q}\right)^{2}+b \log U_{e q}+c U_{e q}+d=0
$$
avec:

$$
\begin{aligned}
& a=\frac{m}{16} \\
& b=-\frac{m}{8}\left\{\frac{4}{m \sigma_{c}} \sqrt{\sigma_{c}^{2}-m \sigma_{c} \sigma_{y}}\right. \\
& \left.+\log \left(\frac{0,75}{E} \sqrt{\sigma_{c}^{2}-m \sigma_{c} \sigma_{y}}\right)\right\} \\
& c=K_{s} \frac{a^{s}\left(d_{0}\right)-1}{\sigma_{c}} \\
& d=\frac{m}{16}\left\{\frac{4}{m \sigma_{c}} \sqrt{\sigma_{c}^{2}-m \sigma_{c} \sigma_{y}}\right. \\
& \begin{array}{c}
\left.+\log \left(\frac{0,75}{E} \sqrt{\sigma_{c}^{2}-m \sigma_{c} \sigma_{y}}\right)\right\}^{2} \\
\sigma_{y}=-\frac{m \sigma_{c}}{8}-P_{m}+\frac{\sigma_{c}}{2} \sqrt{\frac{m^{2}}{16}+\frac{m P_{s}}{\sigma_{c}}+1}
\end{array} \\
& \begin{array}{c}
\left.+\log \left(\frac{0,75}{E} \sqrt{\sigma_{c}^{2}-m \sigma_{c} \sigma_{y}}\right)\right\}^{2} \\
\sigma_{y}=-\frac{m \sigma_{c}}{8}-P_{m}+\frac{\sigma_{c}}{2} \sqrt{\frac{m^{2}}{16}+\frac{m P_{s}}{\sigma_{c}}+1}
\end{array}
\end{aligned}
$$

e) Plasticité Hoek-Brown Modifié (pour $\mathrm{P}_{\mathrm{eq}}<\left|\sigma_{\mathrm{y}}\right|$. sinon le massif est élastique):

$$
A_{1}\left(B_{1} \log U_{e q}+C_{1}\right)^{1 /(1-a)}+D_{1} U_{e q}+E_{1}=0
$$

avec:

$$
\begin{aligned}
\mathrm{A}_{1}= & \frac{1}{\mathrm{~m}_{\mathrm{b}}} \\
\mathrm{B}_{1}= & \frac{(\mathrm{a}-1)}{2} \mathrm{~m}_{\mathrm{b}} \\
\mathrm{C}_{1}= & \frac{(\mathrm{a}-1)}{2} \mathrm{~m}_{\mathrm{b}} \log \left\{\frac{\mathrm{E}}{0,75} \frac{1}{\sigma_{\mathrm{c}}}\left(-\mathrm{m}_{\mathrm{b}} \frac{\sigma_{\mathrm{y}}}{\sigma_{\mathrm{c}}}\right)^{-\mathrm{a}}\right\} \\
& +\left(-\mathrm{m}_{\mathrm{b}} \frac{\sigma_{\mathrm{y}}}{\sigma_{\mathrm{c}}}\right)^{1-\mathrm{a}} \\
\mathrm{D}_{1}= & \mathrm{K}_{\mathrm{s}} \frac{\mathrm{a}^{\mathrm{s}}\left(\mathrm{d}_{0}\right)-1}{\sigma_{\mathrm{c}}} \\
\mathrm{E}_{1}= & U_{\mathrm{f}} \mathrm{K}_{\mathrm{s}} \frac{\left(1-\mathrm{a}^{\mathrm{s}}\left(\mathrm{d}_{0}\right)\right)}{\sigma_{\mathrm{c}}}
\end{aligned}
$$

Et $\sigma_{y}$ donnée par :

$$
\frac{\sigma_{c}}{2}\left(-m_{b} \frac{\sigma_{y}}{\sigma_{c}}\right)^{a}-\sigma_{y}-P_{\infty}=0
$$

\section{Annexe 2}

a) Domaine de validité de la "Nouvelle Méthode Implicite " en viscoplasticité:

$$
\mathrm{V}^{*} \leq 5000, \quad 1<\mathrm{N}_{\mathrm{s}} \leq 5
$$

b) Paramètres $A$ et $B$ fonctions de $N_{5}$ et de $V^{*}$

$A\left(V^{*}\right)=0,018+0,982 \exp \left(-\left(\log \left(1+V^{*}\right) / 2,8\right)^{6,4}\right)$

$2 \mathrm{~B}\left(\mathrm{~N}_{\mathrm{s}}, \mathrm{V}^{*}\right)=b_{02}\left(\mathrm{~V}^{*}\right) \cdot\left(\mathrm{p}_{1}\left(\mathrm{~N}_{\mathrm{s}}\right)+\left(1-\mathrm{p}_{1}\left(\mathrm{~N}_{\mathrm{s}}\right)\right) \exp (-\log (1+\right.$ $\left.\left.\left.\left.V^{*}\right) / 2,3\right)^{3,3}\right\}\right)$

où : $b_{02}\left(V^{*}\right)=0,195+1,805 \exp \left(-\left(\log \left(1+V^{*}\right) / 2,71\right)^{4,84}\right)$

$$
p_{1}\left(N_{s}\right)=\exp \left(1,756-0,878 N_{s}\right)
$$

c) Convergences au front de taille:

Tunnel non soutenu:

$\mathrm{U}_{1}\left(\mathrm{~N}_{\mathrm{s}}, \mathrm{V}^{*}\right) / \mathrm{U}_{-}=\mathrm{p}_{2}\left(\mathrm{~N}_{\mathrm{s}}\right)+\left(0,282-\mathrm{p}_{2}\left(\mathrm{~N}_{\mathrm{s}}\right)\right)$

$\exp \left(-\left(\log \left(1+V^{*}\right) / 2,1\right)^{2,15}\right)$

Tunnel soutenu:

$\mathrm{U}_{\mathrm{f}}\left(\mathrm{N}_{\mathrm{s}^{\prime}}, \mathrm{V}^{*}\right) / \mathrm{U}_{\infty}=\mathrm{p}_{2}\left(\mathrm{~N}_{\mathrm{s}}\right)+\left(0,413-0,0627 \mathrm{~N}_{\mathrm{s}}-\mathrm{p}_{2}\left(\mathrm{~N}_{\mathrm{s}}\right)\right)$ $\exp \left(-\left(\log \left(1+V^{*}\right) / 2,7\right)^{2,9}\right)$

où : $p_{2}\left(N_{s}\right)=0,27 N_{s} / \exp \left(N_{s}-1\right)$

d) Coefficients $C_{1}$ et $C_{q}$ fonctions de $N_{5}$ et de $V^{*}$ :

$C_{1}\left(N_{s}, V^{*}\right)=p_{c 1}\left(N_{s}\right)-\left(p_{c 1}\left(N_{s}\right)-0,5\right) \exp (-(\log (1+$ $\left.\left.\mathrm{V}^{*}\right) / 3,54\right) \mathrm{m}_{\mathrm{ct}}^{\left.(\mathrm{N} s)^{2}\right)}$

où: $p_{\mathrm{c} 1}\left(\mathrm{~N}_{\mathrm{s}}\right)=\exp \left(-0,118+0,817 \mathrm{~N}_{\mathrm{s}}\right)$

$\mathrm{m}_{\mathrm{c} 1}\left(\mathrm{~N}_{\mathrm{s}}\right)=\exp \left(2,094+0,0467 \mathrm{~N}_{\mathrm{s}}\right)$

$\mathrm{C}_{2}\left(\mathrm{~N}_{\mathrm{s}^{\prime}} \mathrm{V}^{*}\right)=\mathrm{P}_{\mathrm{c} 2}\left(\mathrm{~N}_{\mathrm{s}}\right)-\left(\mathrm{p}_{\mathrm{c} 2}\left(\mathrm{~N}_{\mathrm{s}}\right)-0,6\right) \exp (-\mathrm{log}(1+$ $\left.\left.\mathrm{V}^{*}\right) / 3,7\right) \mathrm{m}^{\left({ }^{(N)}\right)}$ )

où: $p_{c 2}\left(N_{s}\right)=\exp \left(-0,713+1,172 N_{s}\right)$

$$
\mathrm{m}_{\mathrm{c} 2}\left(\mathrm{~N}_{\mathrm{s}}\right)=-0,564+2,51 \mathrm{~N}_{\mathrm{s}} \text {. }
$$

